\title{
Analysis of Temporal and Structural Characteristics of Jovian Radio Emissions for Passive Radar Sounding of Jupiter's Icy Moons
}

\author{
Leonardo Carrer, Member, IEEE, Dustin M. Schroeder, Senior Member, IEEE, Andrew Romero-Wolf, Paul Ries,
} Lorenzo Bruzzone, Fellow, IEEE

\begin{abstract}
Recent studies have proposed that Jovian Decametric Radiation (DAM) can be effectively exploited for probing the subsurface of Jupiter's icy moons by using passive radio sounding. However, these studies were based on the assumption that Jovian noise is white and stationary. Therefore, additional investigations into the temporal stability, predictability, and spectral properties of Jovian noise are required to fully assess the potential of passive radar sounding and improve the acquisitions planning. In this paper, we investigate these properties of the Jovian DAM to understand their impact on radar sounding performance. This is done by analyzing the recently available radio spectra acquired by the JUNO Waves instrument. Results are also evaluated for the specific case of the Radar for Icy Moon Exploration (RIME) and the Radar for Europa Assessment and Sounding: Ocean to Near-surface (REASON) that have been selected for ESA and NASA missions to Ganymede and Europa. Our results show that the Jovian DAM is not perfectly white but no severe distortions in the range response should be expected. The results on spatio-temporal occurrence show that Jupiter's DAM has a variable probability of occurrence, which is rather sporadic for some frequency ranges. The results on RIME and REASON flybys show that the Jovian DAM occurrence probability is relatively low for selected sub-jovian flybys at Europa. For the RIME Ganymede orbital phase a large number of high occurrence passive acquisition opportunities are expected. The experimental results also show that a large bandwidth receiver would enhance the chance of recording Jovian noise.
\end{abstract}

Index Terms-Passive Radar, Radar Sounder, Jupiter Icy Moons, Jovian DAM, JUNO, CML, Io phase.

\section{INTRODUCTION}

$\mathbf{R}$ ADAR sounders are airborne or spaceborne electromagnetic sensors operating at relatively low frequencies in the HF/VHF part of the spectrum (i.e. MHz range). As the radar sounder transmitted electromagnetic waves travel through the subsurface, each dielectric discontinuity in the probed medium results in part of the incoming signal being reflected towards the radar. These radar echoes are subsequently recorded by the sensor. By analyzing them it is possible to obtain crucial information on the subsurface structure and composition. This characteristic makes radar sounders valuable instruments for the subsurface exploration of the Earth and other planetary bodies [1][2][3][4].

L. Carrer and L. Bruzzone are with the Department of Information Engineering and Computer Science, University of Trento, Trento, Italy.

D. M. Schroeder is with the Department of Geophysics and with the Department of Electrical Engineering, Stanford University, Stanford, CA, USA

A. Romero-Wolf and P. Ries are with the Jet Propulsion Laboratory, California Institute of Technology, Pasadena, CA, USA.
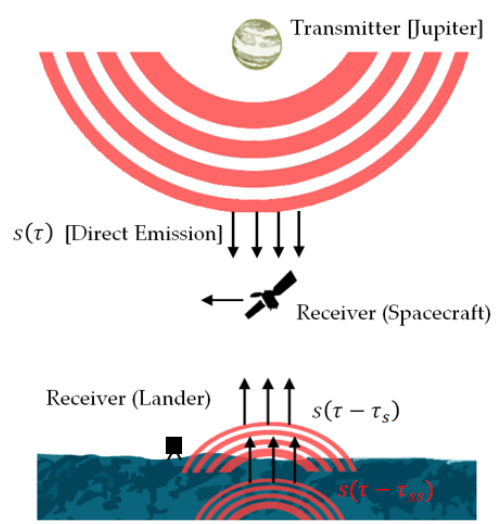

Fig. 1: Illustration of passive subsurface sounding using Jovian Decametric Radiation. $s(\tau)$ is the transmitted signal from Jupiter while $s\left(\tau-\tau_{s}\right)$ and $s\left(\tau-\tau_{s s}\right)$ are the echoes from the surface and subsurface respectively.

Jupiter's icy moons (i.e. Europa, Ganymede and Callisto) are compelling targets for radar subsurface investigations as there is indirect evidence of the presence of a global oceans underneath their surface and of possible water pockets in the shallow subsurface [5]. In this context, two radar sounders namely the Radar for Icy Moon Exploration (RIME) [6] and the Radar for Europa Assessment and Sounding: Ocean to Near-surface (REASON) [7] are planned to sound Ganymede (orbit phase and flybys, respectively) and Europa (flybys). RIME will also perform a limited number of flybys on Callisto and Europa. Currently, both active radars are planning to cover the radio occulted regions (i.e, anti-jovian side) of the icy moons due to Jupiter's strong radio emissions. RIME operates at $9 \mathrm{MHz}$ (High-Frequency (HF) band) central frequency with bandwidth of about $3 \mathrm{MHz}$, while REASON operates at both $9 \mathrm{MHz}$ and $60 \mathrm{MHz}$ (Very High-Frequency (VHF) band) central frequencies with bandwidth of $1 \mathrm{MHz}$ and $10 \mathrm{MHz}$, respectively. As a result of the multi-frequency capability, REASON VHF (60 MHz) sub-jovian acquisitions are planned as this frequency band is clear of Jupiter noise emissions.

Jupiter is one of the most powerful sources of radio waves at frequencies below about $40 \mathrm{MHz}$ [8]. In particular, the range from $3 \mathrm{MHz}$ to the upper-cutoff frequency of $41 \mathrm{MHz}$ is defined as Decametric radiation (DAM). DAM radio emissions originate from the interaction between strong magnetic field 
of Jupiter and the magnetospheric plasma [9].

Romero-Wolf et al. proposed that Jovian DAM could be exploited for passive radio sounding of the subsurface of Europa, Ganymede and Callisto from orbit [10] or from a lander platform [11]. The principle of operation of a passive sounder is the following. The radar sounder antenna records both the radio emission from Jupiter and its reflections from the subsurface. Correlation of the direct emission and reflected signals result in estimation of delays and amplitudes of the echoes (see Fig. 1).

The Jovian DAM frequency range is particularly suitable for passive radar acquisitions as (i) it is above the Europa's ionospheric cut-off frequency [12], and (ii) its larger exploitable bandwidth with respect to jovian kilometric radiation which results in improved resolution of subsurface structures. Even if the Jovian DAM emissions above the ionospheric cutoff frequency, the presence of ionospheric distorting effects should be taken into account. The severity of these effects is function of the emission frequency and is expected to be more prominent at low frequencies [12], [13].

Jovian burst densities at Jupiter's icy moons are expected to be in the order of $\left[10^{-14}, 10^{-16}\right] \mathrm{W} \mathrm{m}^{-2} \mathrm{~Hz}^{-1}$ (see Fig. 2 ). These estimated values are consistently higher than the galactic background radiation [8], [14]. Schroeder et al. [15] showed that the addition of a passive sounding mode on RIME or REASON, under given assumptions, would enhance their science return by enabling sub-Jovian HF sounding in the presence of decametric Jovian noise. REASON VHF band is unaffected by the Jovian DAM interference, but its subsurface penetration capability is limited with respect to HF band [16]. Thus, recently a passive low frequency (about $1 \mathrm{MHz}$ ) instrument has been proposed [17]. Recently, Gerekos et al. [18] presented a method for simulating and comparing both passive and active acquisitions of jovian icy moons.

Radio observation from Earth revealed that the probability of occurrence of decametric emission is correlated with the Central Meridian Longitude (CML) of Jupiter and with the orbital position of the moon Io with respect to superior geocentric conjunction [9], [19] denoted as $\varphi_{I o}$. Accordingly, the probability of having a Jovian radio emission and thus, a potential passive acquisition, depends on the particular value of the $C M L$ and $\varphi_{I o}$ computed with respect to the observer position (see Fig. 3 (a)). By using ground-based and spacecraft observations, radio astronomers have identified four main regions in the $C M L$ vs $\varphi_{I o}$ where the radiation occurrence is relatively high and are denoted as Io regions and marked with the letter from A to D [9]. The letter order roughly reflects the likelihood of observing DAM radiation in that particular part of the CML vs Io phase plane. Certain jovian emissions are not controlled by Io and are denoted as non-Io emissions. Also these emissions are marked with letters from $\mathrm{A}$ to $\mathrm{D}$ and can overlap in the $C M L$ and $\varphi_{I o}$ with the Io controlled ones. The main regions are depicted in Fig. 3 (b).

The spatial, temporal occurrence, and predictability of the Jovian Decametric Radiation, which is required for sub-jovian HF passive and active acquisition planning, is still poorly understood in the literature even though there have been many observations from Earth since its discovery in 1955 [20].
This is due to the fact that (i) there is a modest availability of in-situ collected data and (ii) ground observations from Earth are limited by the terrestrial ionosphere, which prevents observations at frequencies below $10 \mathrm{MHz}$ [21]. Moreover, all the previous passive sounding studies approximated the Jovian Decametric Radiation as white noise [10], [15]. Any non-white frequency content in the Jovian radio signal could compromise unambiguous interpretation of the subsurface features.

In 2016, the JUNO probe began to acquire extensive measurements of the Jovian DAM emissions at different frequencies with the JUNO Waves [22] instrument. The recently available data offer the opportunity to perform an extensive characterization of Jovian DAM across the frequency range between $3 \mathrm{MHz}$ and $41 \mathrm{MHz}$. Indeed, the thorough understanding of the Jovian DAM emissions properties based on both theoretical and experimental analyses potentially have significant implications for both active and radar sounding of Jupiter icy moons. It would allow to (i) understand the actual performance of passive sounding as function of the emission frequency (ii) schedule acquisitions for both active and passive mode and (iii) evaluate the actual performance of RIME and REASON when operating as passive radars and the likelihood of having a successful passive acquisition.

In this paper, we first theoretically characterize the general properties of the Jovian decametric radiation as a subsurface sounding signal of opportunity with particular emphasis on its autocorrelation properties. Then, we experimentally assess, by exploiting JUNO Waves data, the spectral characteristics of Jovian Decametric Radiation, its temporal occurrence and suitability as potential subsurface sounding signal as a function of the radio emission frequency. We also derive statistics on the expected flux density in the proximity of the moons.

The paper is organized as follows. In Section II we introduce the principles of subsurface echo detection for passive radar sounder and describe the main factors affecting performance such as the autocorrelation spectral structure and the timebandwidth product. Section III presents the results of the analysis of JUNO experimental data regarding Jovian noise spectral structure, temporal occurrence, power levels and capability of prediction. The results presented from Section III-B to III-D have general validity and cover different Jovian DAM emission frequency ranges (between $3.5 \mathrm{MHz}$ and $19.5 \mathrm{MHz}$ ). Section III-F is devoted to the specific analysis of RIME and REASON passive performance and to the related implications on operations resulting from the JUNO data. Finally, Section IV and Section V provide discussion of the results and the conclusions of the paper, respectively.

\section{Principles of Subsurface Echo Detection and WAVEForm DESIGN FOR PASSIVE RADAR SOUNDERS}

In this section we describe the main components governing the passive radar subsurface echo detection capabilities. In particular, we analyze the properties of the autocorrelation of the subsurface probing waveform which determines the point spread function of the passive radar. To better understand the passive radar subsurface echo detection mechanism, we highlight the similarities and differences with respect to an 


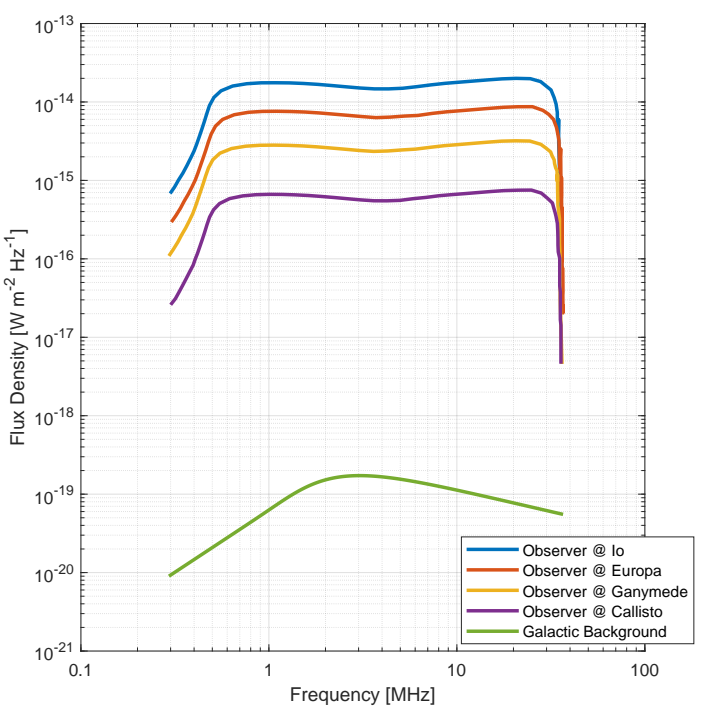

Fig. 2: Estimated flux densities of Jovian DAM for different Icy Moons [8]. The Jovian DAM emission levels are substantially higher than the galactic background flux density computed as in [14].

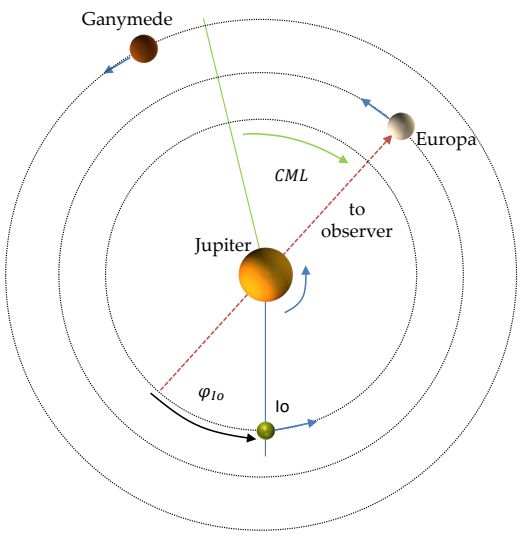

(a)

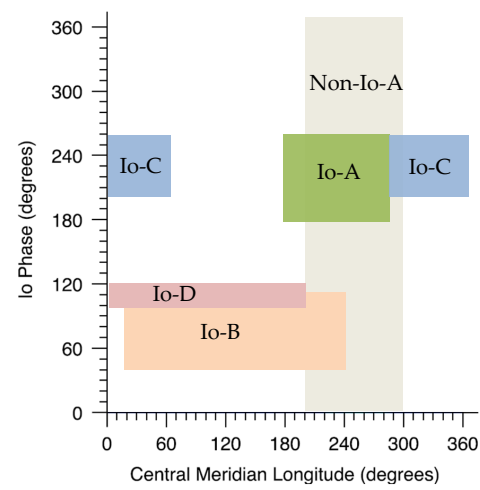

(b)

Fig. 3: (a) Definition of the observer Central Meridian Longitude $C M L$ and Io phase $\varphi_{I o}$. In this example, the observer is positioned on Europa. (b) Definition of the main regions on the $C M L$ vs $\varphi_{I o}$ plane where the Jovian DAM has higher occurrence probability [9]. active radar, where the transmitted waveform is not a signal of opportunity.

Radar sounders infer the distance of a given surface or subsurface target by estimating its respective echo time delays. A common method for echoes delay estimation is the computation of the cross-correlation between the transmitted signal $s(\tau)$ and the received signal $r(\tau)$, where $\tau$ is an arbitrary time. Typically the transmitted signal is linear frequency modulated (i.e, chirp). The time delay of a given surface or subsurface reflector, appearing as a peak in the cross-correlation output, is then converted to depth by knowing the electromagnetic wave velocity in the medium and the radar sampling rate. The received signal $r(\tau)$ is the summation of all the radar echoes which are amplitude scaled and time shifted versions of the transmitted signal $s(\tau)$. Let us assume a two-reflection model where $\sqrt{P_{s}} s\left(\tau-\tau_{s}\right)$ and $\sqrt{P_{s s}} s\left(\tau-\tau_{s s}\right)$ are the signals reflected from the surface and subsurface of a given planetary body and recorded at time delays $\tau_{s}$ and $\tau_{s s}$ with echo power equal to $P_{s}$ and $P_{s s}$ respectively (see Fig. 1). In the active radar case, the cross-correlation $R_{a}(t)$ is equal to:

$$
R_{a}(t)=\sqrt{P_{s} P_{t, a}} \hat{R}_{s}\left(t-\tau_{s}\right)+\sqrt{P_{s s} P_{t, a}} \hat{R}_{s}\left(t-\tau_{s s}\right)
$$

where $P_{t, a}$ is the active radar transmitted power and $t$ is the autocorrelation time (i.e., variable time lag). The autocorrelation function $R_{s}(t)$ is equal to:

$$
R_{s}(t)=[s(\tau) \otimes s(\tau)](t)=\frac{1}{T_{s}} \int_{-T_{s} / 2}^{T_{s} / 2} s(t+\tau) s^{*}(\tau) d \tau
$$

where $T_{s}$ is the integration time equal to $T_{a}$ and $T_{p}$ for the active and passive radar respectively. In the active case, $T_{a}$ is a-priori determined by the radar system engineer (i.e. pulse length). In the passive case, $T_{p}$ is bounded by either the time duration of the random source or by the duration of the acquisition window. We define as $\hat{R}_{s}(t)$ the normalized autocorrelation function equal to:

$$
\hat{R}_{s}(t)=\frac{R_{s}(t)}{R_{s}(0)}
$$

Alternatively, the autocorrelation function of the transmitted signal can be derived, according to Wiener-Khinchin theorem, from its power spectral density denoted as $S(f)$ in the following way:

$$
R_{s}(t)=\mathcal{F}^{-1}\{S(f)\}
$$

where $\mathcal{F}^{-1}\{\cdot\}$ is the inverse Fourier transform operator. In the passive radar case, the transmitted signal $s(\tau)$ is recorded along with the surface and suburface reflections. The autocorrelation $R_{p}(t)$ is equal to [10]:

$$
\begin{aligned}
R_{p}(t)= & \left(P_{t, p}+P_{s}+P_{s s}\right) \hat{R}_{s}(t)+\sqrt{P_{s} P_{t, p}} \hat{R}_{s}\left(t-\tau_{s}\right)+ \\
& +\sqrt{P_{s s} P_{t, p}} \hat{R}_{s}\left(t-\tau_{s s}\right)+\sqrt{P_{s} P_{s s}} \hat{R}_{s}\left(t-\tau_{s s}+\tau_{s}\right)
\end{aligned}
$$

where $P_{t, p}$ is the Jovian noise power (i.e. opportunity signal). For both the active and passive radar sounders [see (1) and (5)] the received echo trace, after the correlation procedure, is a superposition of scaled and shifted replicas of the autocorrelation function $R_{s}(t)$. If the incident field is recorded separately from the reflected field, then the passive radar autocorrelation 
becomes equal to the cross-correlation of the active radar (1). However, this requires two directional antennas, one pointing at the radiation source and one at the target area respectively.

The shape of $R_{s}(t)$ (i.e. correlation properties of $s(t)$ ) is of fundamental importance in radar sounding. Its main lobe width impacts the range resolution of the radar and its side lobes the ability of detecting the weak subsurface targets. A schematic representation of the autocorrelation function $R_{s}(t)$ and the typical response after correlation for an active (i.e. $R_{a}(t)$ ) and passive (i.e. $\left.R_{p}(t)\right)$ radar for the two reflections model is shown in Fig. 4. For the active radar sounding case, $s(t)$ is assumed to be a linear frequency modulated signal $s_{a}(t)=\exp \left(j \pi B / T_{a} t^{2}\right)$ where $B$ is the signal bandwidth. We denote as $\Omega$ the time-bandwidth product:

$$
\Omega=B T_{s}
$$

For large values of $\Omega$, the power of the normalized autocorrelation function of $s(t)=s_{a}(t)$ is equal to $\left|\hat{R}_{s}(t)\right|^{2}=$ $|\operatorname{sinc}(\pi B t)|^{2}$ [see (2)]. The range resolution is proportional to $1 / B$ and the side lobe structure is given by the sinc shape. The value of $\Omega$ is a-priori determined by the radar system engineer by defining the transmitted radar waveform bandwidth $B$ and pulse duration $T_{s}=T_{a}$ of the linear frequency modulated signal. In particular, the value of $T_{a}$ is generally a trade-off with respect to the allowable transmitted pulse power in a given time frame due to technological limitations.

In the passive radar sounding case, it is assumed that $s(t)=n(t)$ where $n(t)$ is stationary white gaussian process. In principle, the autocorrelation function $R_{s}(t)$ of gaussian white noise is a Dirac delta function. This occurs assuming an infinite bandwidth $B$ and integration time $T_{s}$ [23]. In practical cases, both $B$ and $T_{s}$ are limited. This results in range resolution proportional to $1 / B$ where $B$ is the rectangular lowpass filter bandwidth applied to the white noise. The value of the signal bandwidth $B$ should be selected accordingly to the bandwidth of the random source. The low-pass filter shapes the response as $|\operatorname{sinc}(\pi B t)|^{2}$. The integration time $T_{s}=T_{p}$ is mainly determined by the acquisition geometry as it will be described in the next section. The random noise contribution results in a noise floor, with respect to the peak of the autocorrelation, equal to $2 \Omega$ [23], [24]. This is intended as mean value. Thus, it is crucial to select proper values of $\Omega$ in order to detect the weak subsurface reflections which otherwise will be dominated by the noise floor. An example of the autocorrelation function of filtered white noise (mean and standard deviation) for different integration time is shown in Fig. 5. Arguably, the noise floor effect can be drastically reduced assuming that the passive integration time $T_{s}=T_{p}$ could be substantially larger than the active case $\left(T_{s}=T_{a}\right)$ for suitable geometries and temporal stability of the random source. Typical values of $T_{a}$ for planetary radar sounding are in the order of $100 \mu \mathrm{s}$ [2]. Finally, both in the active and passive radar, there is an increase of the signal-to-noise ratio after the correlation procedure by a factor proportional to $\Omega$ which is denoted as integration gain. In the next section, we describe and discuss the factors affecting the value of $\Omega$ in the passive radar case.
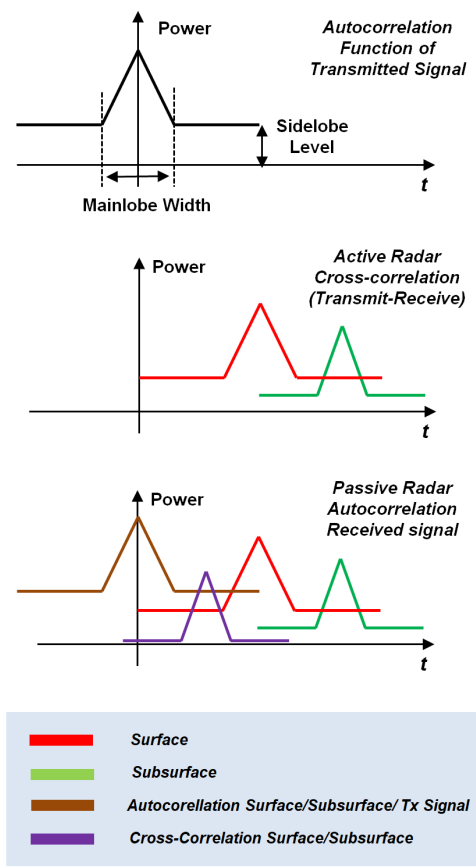

Fig. 4: Illustration of cross correlation for active and passive radar. When compared to the active case, in the passive cross correlation case there are more terms (brown and purple) due to the fact that the transmitted signal is simultaneously recorded with the surface and subsurface reflections.

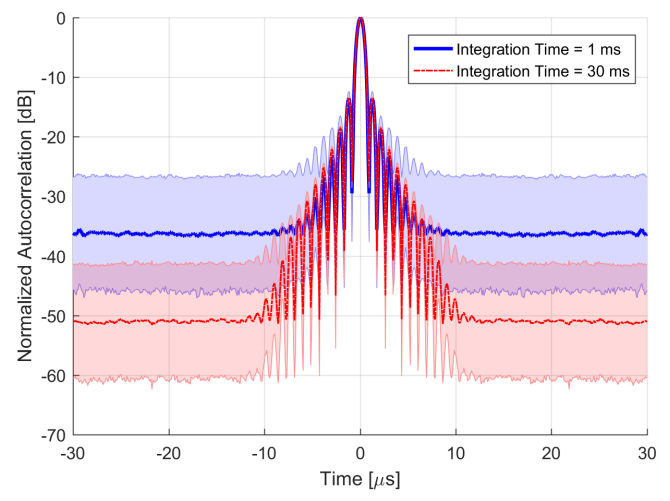

Fig. 5: Example of the power of the passive radar ideal autocorrelation function $\left|\hat{R}_{s}(t)\right|^{2}$ versus time (mean and standard deviation) assuming a variable integration time $T_{s}$ and a fixed bandwidth $B \simeq 1$ $\mathrm{MHz}$. The number of random waveform used to compute statistics is equal to 2000 for each experiment.

\section{A. Passive Radar Sounder Performance Dependence on Time- Bandwidth Product}

In this section, we derive the lower and upper bounds for the passive radar time-bandwidth product for determining its actual sensitivity to subsurface echoes.

The integration time $T_{s}$ is limited by the time the target remains within the first Fresnel zone [15]:

$$
T_{s} \leqslant \frac{2 \sqrt{\lambda h}}{v}
$$

where $h$ and $v$ are the sensor height and velocity respectively 
and $\lambda$ the signal of opportunity wavelength. The bandwidth $B$ cannot exceed twice the central frequency such that:

$$
B \leq \frac{2 c_{0}}{\lambda}
$$

By assuming that $\lambda=2 c_{0} / B$, the maximum time-bandwidth product $\Omega$ is equal to:

$$
\Omega \leq \frac{2 \sqrt{\lambda h}}{v} B=\frac{2 \sqrt{2 c_{0} h}}{v} \sqrt{B}
$$

This implies that for a passive radar sounder, the integration time (which depends on $v$ ) plays a more important role in the determination of $\Omega$ when compared to the bandwidth. By assuming a sensor in circular orbit, the maximum integration time is in the order of seconds for both the Europa and Ganymede (see Fig. 6) (a). The resulting value of the integration gain is shown in Fig. 6 (b), which confirms the fact that the bandwidth impact on noise floor is marginal with respect to the integration time. It is important to state that the results of (9) are obtained considering a bandwidth value which is currently not achievable due to technological limitations. At the current status, a feasible value for the maximum bandwidth is $B=c_{0} /(2 \lambda)$. To overcome the integration time limitations [see (7)], a passive lander can be deployed which does not have any theoretical constraint on $T_{s}$ [11].

The minimum value of $\Omega$ for subsurface detection can be derived from the following analysis. In the passive case, the correlated signal $R_{p}(t)$ [see (5)] has a strong peak equal $P_{t, p}+$ $P_{s}+P_{s s}$ for $t=0$. In order to detect the weak subsurface reflections, the noise floor level of the reflection at $t=0$ should be sufficiently small. This dictates a minimum value on the time-bandwidth product $\Omega$. The transmitted power $P_{t, p}$ is equal to [15]:

$$
P_{t, p}=\frac{N_{0} \lambda^{2}}{4 \pi} B
$$

where $N_{0}$ is the environmental radio noise power $\left(W H z^{-1} m^{-2}\right)$. By assuming that $P_{t, p} \gg P_{s s}, P_{s}$ [see (5)], the detection of a given subsurface interface occurs when the following condition is met:

$$
\frac{P_{t, p}}{2 \Omega}<P_{s s}
$$

where $P_{s s}$ is the echo power of the subsurface signal. The above expression and the model formulation hereafter is valid under the assumption that surface off-nadir echoes power (i..e, clutter) is negligible with respect to the subsurface echoes power. The subsurface echo power can be expressed as:

$$
P_{s s}=\frac{P_{s}}{L_{s s}}
$$

where $P_{s}$ is the surface echo power and $L_{s s}$ are the overall two-way subsurface losses. The surface echo power is equal to [15]:

$$
P_{s}=\frac{P_{t, p}}{\left(1+\sqrt{\frac{h}{\lambda}} \tan \left(\sigma_{s}\right)\right)^{2}}
$$

By substituting (12) and (13) into (11) we obtain that the maximum allowable two-way subsurface losses (i.e., loss budget) depend on $\Omega$ as follows:

$$
L_{s s}<\frac{2 \Omega}{\left(1+\sqrt{\frac{h}{\lambda}} \tan \left(\sigma_{s}\right)\right)^{2}}
$$

where $\sigma_{s}$ is the characteristic slope of the target surface.

According to (14), the maximum value of $L_{s s}$ for which a radar sounder detects a given subsurface interface depends on the geometric parameters, the probing wavelength and the noise characteristics in terms of time-bandwidth product. The value of $\Omega$ is idealized, some margin is needed in practical applications to take into account the sidelobe fluctuations. Given the value of $L_{s s}$ resulting from (14), different penetration depths can be achieved depending on factors such as the assumed subsurface thermal scenario [25]. Fig. 7 reports $L_{s s}$ according to (14) for parametric values of $\Omega, \lambda$ and $\sigma_{s}$. By comparing the results of Fig. 6 (b) and Fig. 7, for $h=500$ $\mathrm{km}$, the maximum subsurface losses as function of $\Omega$ and $\lambda$ are widely compatible with the expected subsurface losses at the icy moons [25].

\section{B. Impact of Waveforms non Idealities on Subsurface Detec- tion Capabilities}

The theoretical results of previous sections are based on the assumption that the passive radar signal is band-pass filtered white noise. In practical cases, any unwanted deviation in the structure of the autocorrelation function $R_{s}(t)$ will impact performance on range resolution and/or on noise floor level depending on the type of introduced signal distortion. According to radar theory, side lobes and resolution performance are affected by rippling variations of the autocorrelation spectrum (i.e. power spectral density) [26]. These rippling variations can affect both the magnitude and phase of the power spectral density. In this analysis, we neglect the phase ripples by assuming a perfect phase matching in the autocorrelation computation.

The nuisance term modeling the spectrum amplitude ripples (denoted as $K(f)$ ) is defined as [26]:

$$
K(f)=\frac{a_{0}}{2}+\sum_{n=-\infty}^{\infty} a_{n} \cos \left(2 \pi c_{n} f\right)
$$

where $a_{n}$ and $c_{n}$ are the generic harmonic amplitude and number, respectively. The distorted power spectral density $\tilde{S}(f)$ is equal to :

$$
\tilde{S}(f)=S(f) \cdot K(f)
$$

where $S(f)$ is the power spectral density defined in (4). In time domain, the distorted autocorrelation function resulting from (16) and denoted as $\tilde{R}_{s}(t)$ is equal to:

$$
\tilde{R}_{s}(t)=R_{s}(t) \otimes\left[\frac{a_{0}}{2} \delta(t)+\sum_{n=-\infty}^{\infty} \frac{a_{n}}{2} \delta\left(t-c_{n}\right)\right]
$$

where $\delta(t)$ is the Dirac function. Let us consider only one harmonic distortion. In this case, the distorted autocorrelation function is equal to:

$$
\tilde{R}_{s}(t)=\frac{a_{0}}{2} R_{s}(t)+\frac{a_{1}}{2} R_{s}\left(t-c_{1}\right)+\frac{a_{1}}{2} R_{s}\left(t+c_{1}\right)
$$



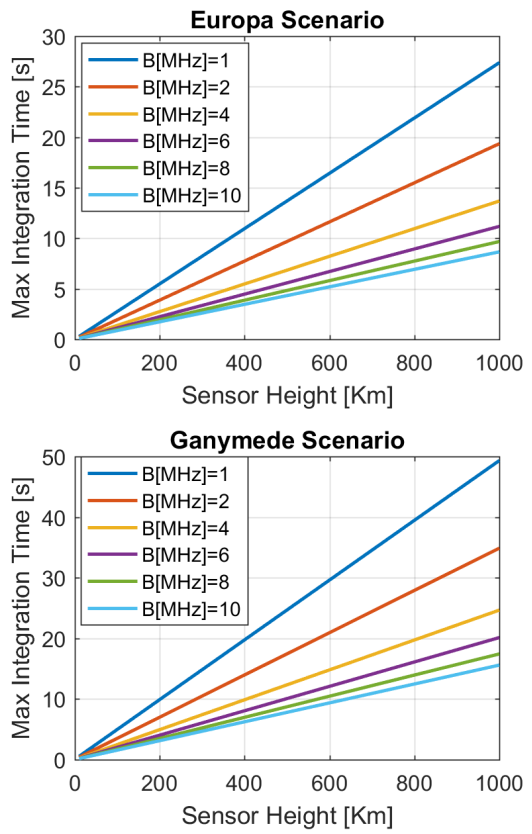

(a)
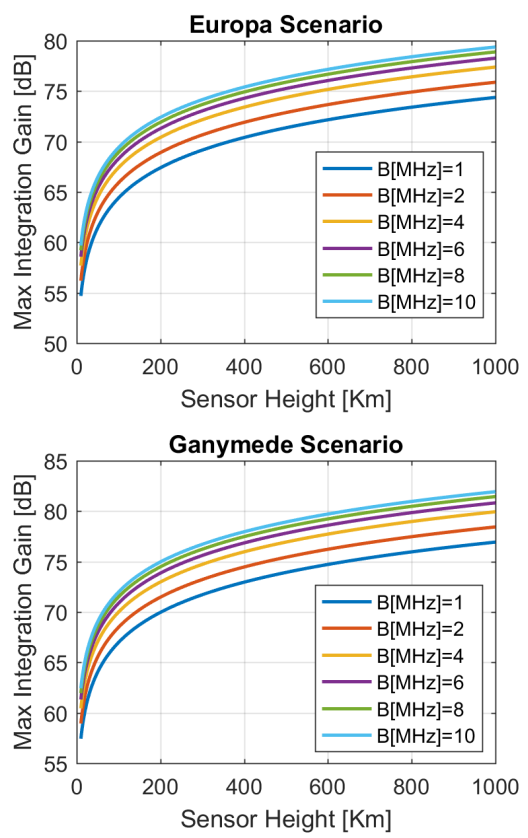

(b)

Fig. 6: (a) Maximum integration time $T_{s}$ for the Europa and Ganymede Scenario orbital sensor case assuming the maximum theoretical limit of $B=2 c_{0} / \lambda$ (b) Maximum Integration gain $10 \log _{10}(2 \Omega)$

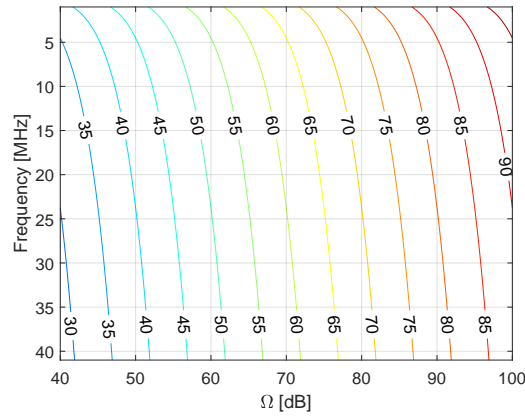

(a)

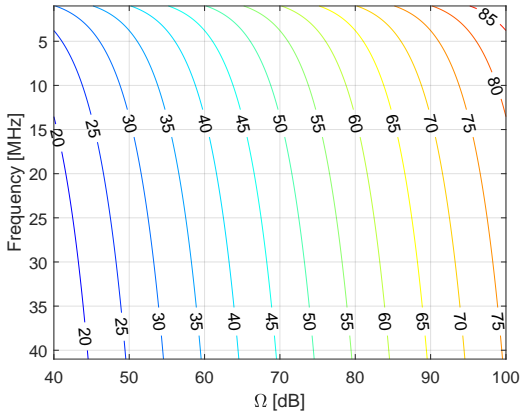

(b)

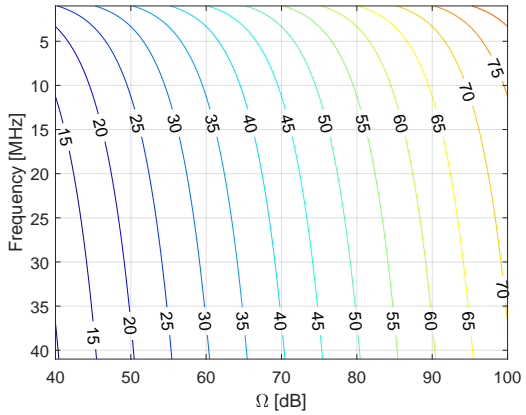

(c)

Fig. 7: Contour plot of the maximum allowable two-way subsurface losses $L_{s s}$ [dB] (i.e, loss budget with no specific assumptions on the subsurface scenario) as function of the probing frequency $c_{0} / \lambda$ and achievable time-bandwidth product $\Omega$ for (a) $\sigma_{s}=1^{\circ}$, (b) $\sigma_{s}=5^{\circ}$ and (c) $\sigma_{s}=10^{\circ}$ and by assuming $h=500 \mathrm{~km}$.

From (18), the autocorrelation distorted output $\tilde{R}_{s}(t)$, is equal to the output of an ideal autocorrelation function $R_{s}(t)$ which is then replicated and centered at time instants equal to the harmonic number $c_{n}$ and amplitude scaled for the harmonic coefficient $a_{n}$. Depending on the values of $a_{n}$ and $c_{n}$, the nuisance term $K(f)$ may degrade resolution (small values of $c_{n}$ ) or increase the overall noise floor level (high values of $\left.c_{n}\right)$. Sufficiently large values of the coefficients $a_{n}$ result in ambiguous subsurface echoes (i.e. artifacts) denoted as paired echoes. In the active radar case, the frequency ripples and any deviations from spectral flatness are generated by the radar electronics. It is common practice in radar system design, to evaluate the aforementioned distortion coefficients from the recorded spectra and to characterize them to minimize their effect on spectral flatness. Passive radar can benefit from a similar approach as will be described in Section III-E.

To give a practical example of the above issues on spectral non idealities for the passive radar case, we evaluated the behavior of the normalized autocorrelation $\hat{R}_{s}(t)$ for different types of colored noise. This is done by assuming a power spectral density that follows a power law of the form [27]:

$$
S(f) \sim \frac{1}{|f|^{\alpha}}
$$

where $\alpha$ is a real number in the interval $[-2,1]$. The following special cases as function of $\alpha$ occur and are of interest for the purpose of this paper:

- $\alpha=0$ corresponds to White noise. 

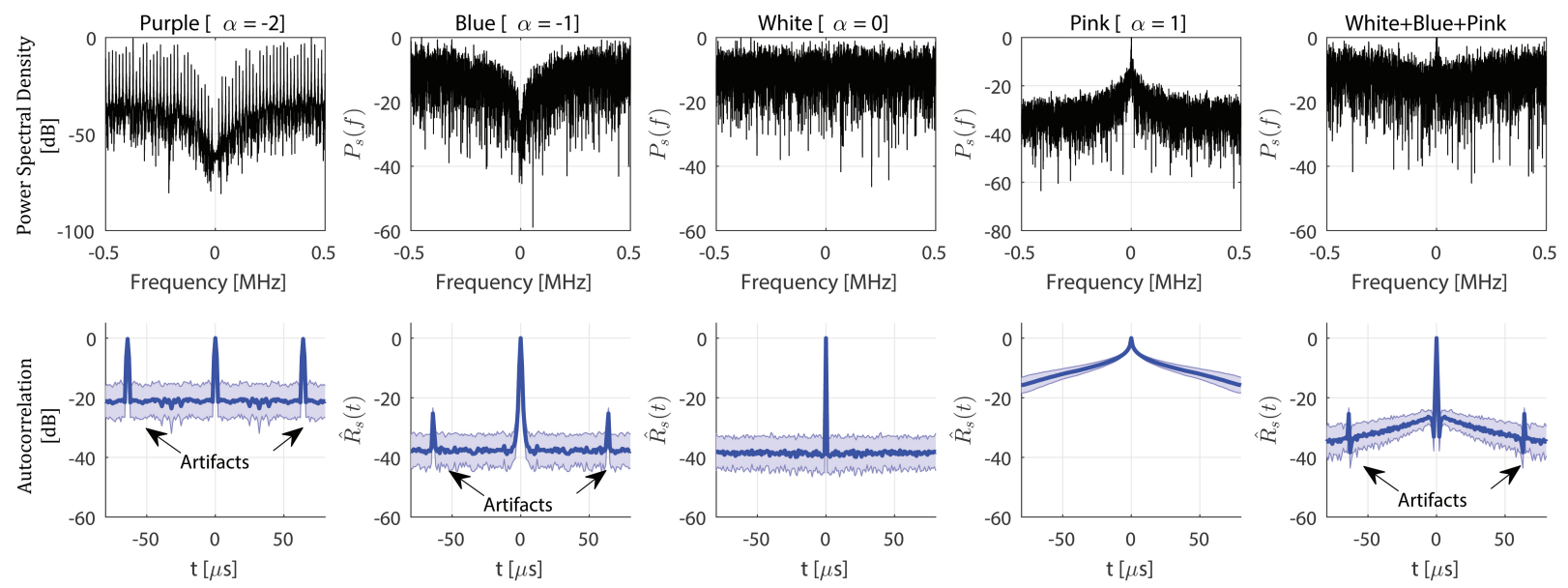

Fig. 8: Power Spectral Density $S(f)$ (example of a single realization) and normalized autocorrelation function $\hat{R}_{s}(t)$ (mean and standard deviation over 100 realizations) for different types of noise generation processes. The generation process is $S(f) \sim 1 /|f|^{\alpha}$ with $\alpha=[-2,1]$. The last plot represents an example of combination of different types of noise. For all the simulations the bandwidth $B$ is equal to $1 \mathrm{MHz}$ and integration time $T_{s}$ equal to $4 \mathrm{~ms}$. The autocorrelation is given by $\hat{R}_{s}(t)=\mathcal{F}^{-1}\{S(f)\}$.

- $\alpha=1$ corresponds to Pink Noise. The power spectral density decreases by $3 \mathrm{~dB}$ per octave.

- $\alpha=-1$ corresponds to Blue Noise. The power spectral density increases by $3 \mathrm{~dB}$ per octave.

- $\alpha=-2$ corresponds to Purple Noise. The power spectral density increases by $6 \mathrm{~dB}$ per octave.

Each type of colored noise introduces artifacts when compared to the white noise case due to the previously described rippling variations in the power spectral density. When compared to white noise, blue noise gives rise to paired echoes and a decrease of the resolution due to the autocorrelation main-lobe increase (see Fig. 8) while pink noise results in an increase in noise floor level. On the other hand, Purple noise power spectral density spikes resemble narrow band interference from the radar electronics. This is also a relevant nuisance which results in an increase in the noise floor level and in the appearance of paired echoes. In the case where other background sources (e.g. galactic noise) do not dominate over the signal of interest (e.g. Jovian DAM), spectral sloping can be corrected or at least mitigated to recover the white noise response by using suitable signal processing techniques as it will be shown in Section III-E.

\section{Experimental ANalysis of the Jovian Noise Spectral and Temporal Structure for Passive SOUNDING}

\section{A. Dataset Description}

The analyses were performed on the Jupiter decametric radiation data acquired by the JUNO Waves instrument [22] from Orbit 00 to Orbit 06 . This is roughly a year-round observation of Jupiter's magnetosphere.

We exploit JUNO Survey and Burst data products [28]. The Survey data contains Jupiter's noise spectrograms (i.e. signal power spectral density as a function of time and frequency) with variable temporal resolution of 1,10 or 30 seconds and fixed spectral resolution of $1 \mathrm{MHz}$. These data products are acquired continuously and divided into acquisition days. An example of a Survey data product is shown in Fig. 9. The Burst data products contain high resolution Jupiter's noise spectrograms with spectral resolution of $320 \mathrm{~Hz}$ spanning $1.3 \mathrm{MHz}$ bandwidth. This corresponds to $3.1 \mathrm{~ms}$ integration time for each realization of the power spectral density. The bandwidth is centered at different emission frequencies for each given burst data product and sampled at the minimum Nyquist rate (i.e. twice the signal bandwidth). When compared to Survey data, these products are recorded for shorter sessions.

The calibrated electric field Survey data are provided in $\mathrm{V}^{2} \mathrm{~m}^{-2} \mathrm{~Hz}^{-1}$ and by assuming an antenna length $L_{\text {eff }}=2.41$ $\mathrm{m}$. This is the geometric effective length of the Juno Waves electric antenna [22]. In electromagnetic waves propagation, a relevant parameter that has to be taken into account is the electrical antenna length, which considers both the geometric length of the antenna and the wavelength of the propagating wave. Sampl et al. [29] determined that the electrical length of the JUNO Waves electric antenna is equal to $L_{\text {eff }}=1.46$ $m$. Kurth et al. [22] estimated the shortest electrical value of the antenna equal to $L_{\text {eff }}=0.46 \mathrm{~m}$. Recent work on whistler mode emissions with JUNO Waves [30] show that $L_{\text {eff }}=1 \mathrm{~m}$ is a reasonable assumption for the value of the antenna length.

Fig. 10 reports the JUNO Waves electric noise levels for different assumptions on the value of $L_{\text {eff }}$. The spectral flux density is proportional to the squared inverse of the effective antenna length $L_{\text {eff }}$. Accordingly, the relative fluxes presented in Fig. 10 were obtained by applying the following scaling factor to the Survey data:

$$
10 \log 10\left(\left(2.41[m] / L_{\text {eff }}\right)^{2}\right)-10 \log 10\left(2 Z_{0}\right)
$$

where $Z_{0}=376.30 \mathrm{ohm}$ is the impedance free space. The normalization (in $\mathrm{dB}$ scale) by $Z_{0}$ is needed to convert the noise levels from $\mathrm{V}^{2} \mathrm{~m}^{-2} \mathrm{~Hz}^{-1}$ to $\mathrm{Wm}^{-2} \mathrm{~Hz}^{-1}$. The Juno noise levels are compared with the power of the galactic background computed from Cane's model [14] (see Fig. 10). 


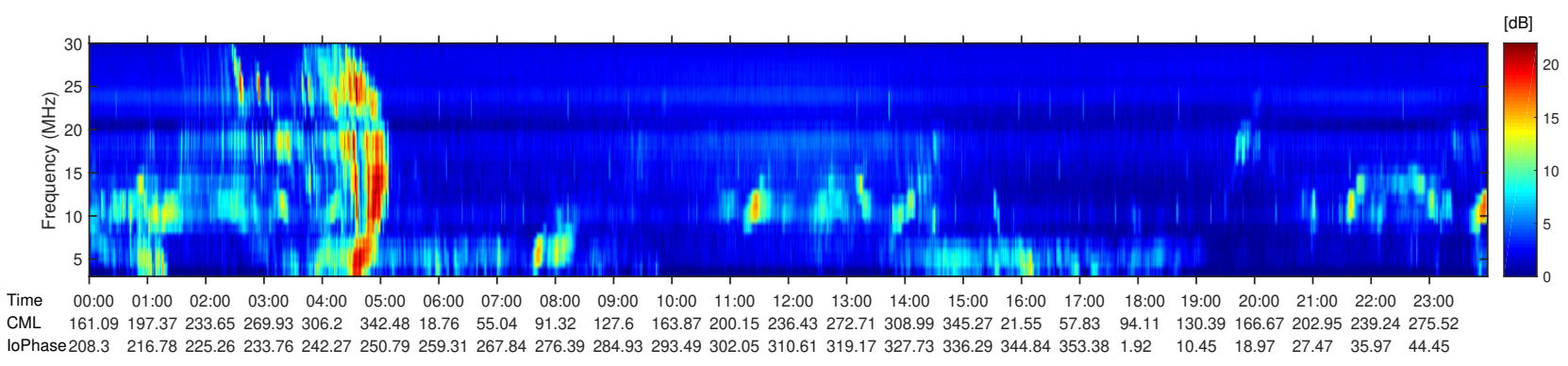

Fig. 9: Example of normalized spectrogram $P \bar{S} D(f, t)$ acquired by JUNO (Survey data product (2017-137). Power Spectral Density values are provided as $\mathrm{dB}$ over background. The acquisition time is correlated with the observer (i.e. JUNO) CML and Io Phase.

Fig. 10 shows that, depending on the antenna effective length $0.46 \mathrm{~m} \leq L_{\text {eff }} \leq 2.41 \mathrm{~m}$, the average JUNO Waves sensitivity in the frequency range under consideration varies from 0 to about $14 \mathrm{~dB}$ above galactic noise. Accordingly, in the following sections, all the experimental results affected by this uncertainty will be provided as a function of the JUNO Waves effective electric antenna length $L_{\text {eff. }}$ Moreover, the instrument is equipped with a variable step attenuator [22] that may reduce its sensitivity. For data considered, the attenuator is always turned off as revealed by the provided data telemetry [28].

By analyzing the results of Fig. 10, we limit our analyses to the frequency range between $3.5 \mathrm{MHz}$ and $19.5 \mathrm{MHz}$, where the JUNO Waves instrument sensitivity is close to the level of the galactic background except for sporadic peaks which are compensated in the data processing stage by notching and interpolation.

We denote each JUNO experimental spectrogram as $P S D(f, t)\left[\mathrm{Wm}^{-2} \mathrm{~Hz}^{-1}\right]$ where $3.5 \mathrm{MHz} \leq f \leq 19.5 \mathrm{MHz}$ and $0 \leq t \leq T$ ( $T$ is the overall time length of the spectrogram). We define the normalized spectrogram $P \bar{S} D(f, t)$ as:

$$
P \bar{S} D(f, t)=\frac{P S D(f, t)}{N_{b}(f)}
$$

where $N_{b}(f)$ is the sensitivity of JUNO at each frequency $f$.

\section{B. Jovian DAM Power levels}

We derived the statistics of the Jovian DAM flux density in the proximity of the icy moons by exploiting JUNO Waves Survey data and by also taking into account the uncertainty on the effective electric antenna length $L_{\text {eff }}$.

The measured JUNO Waves flux densities were re-scaled according to the geometrical spreading factor $\left(D_{\text {juno }} / D_{\text {moon }}\right)^{2}$ where $D_{\text {juno }}$ is the distance of Juno from Jupiter at a given acquisition time and $D_{\text {moon }}$ the average orbital distance of a given icy moon from Jupiter.

Fig. 11 reports the derived average and maximum Jovian DAM power levels for Europa. The error bars represent the uncertainty dictated by the not precise knowledge of $L_{\mathrm{eff}}$ that affects the estimated values. The maximum and average power levels for Ganymede and Callisto can be derived by subtracting $4 \mathrm{~dB}$ and $9 \mathrm{~dB}$, respectively, from the provided figure.
The results confirm the strong intensity of the Jovian DAM in the proximity of the moons. As an example, for the Europa case the peak power levels are in the order of $10^{-14}$ $\mathrm{Wm}^{-2} \mathrm{~Hz}^{-1}$, while the average power level is in the order of $10^{-15.5} \mathrm{Wm}^{-2} \mathrm{~Hz}^{-1}$. We also observe a slightly higher flux density for emission frequencies smaller than $7.5 \mathrm{MHz}$ with respect to higher frequencies. The results on the statistics of the flux density are important to evaluate both the required dynamic range in the case of passive sounding and the signal to interference level in the case of active sounding performed on the Jovian side of a given moon.

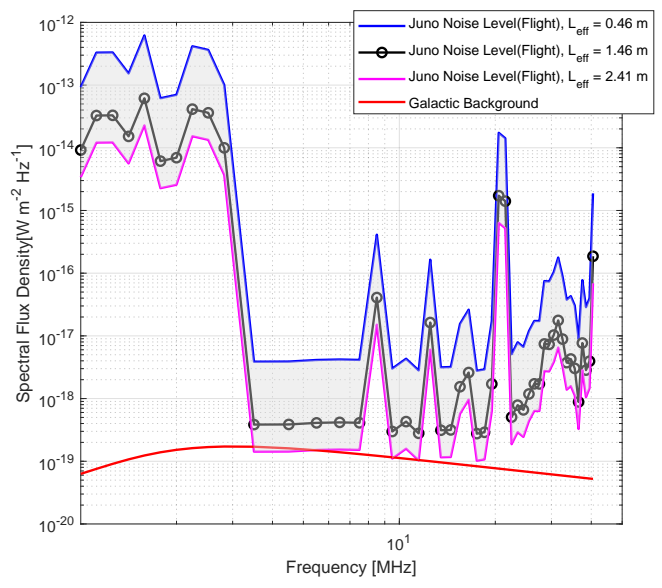

Fig. 10: In-flight JUNO Waves electric noise levels versus frequency compared to galactic background [14] for different effective antenna lengths $L_{\text {eff }}$. The gray area indicates the uncertainty bound with respect to the electrical length of the antenna.

\section{Jovian Noise Spatio-Temporal Occurrence and Predictabil- ity}

This section assesses the Jovian DAM spatial and temporal occurrence as function the Jovian DAM emission frequency. We compiled a Jovian DAM emission catalogue from JUNO Waves Survey data (see example of Fig. 9) spanning the observation dates from day 2016-188 to day 2017-179.

The cataloged emissions are described by their emission frequency $f$ and time duration along with auxiliary data such as Io phase and CML occurring at that specific time. 


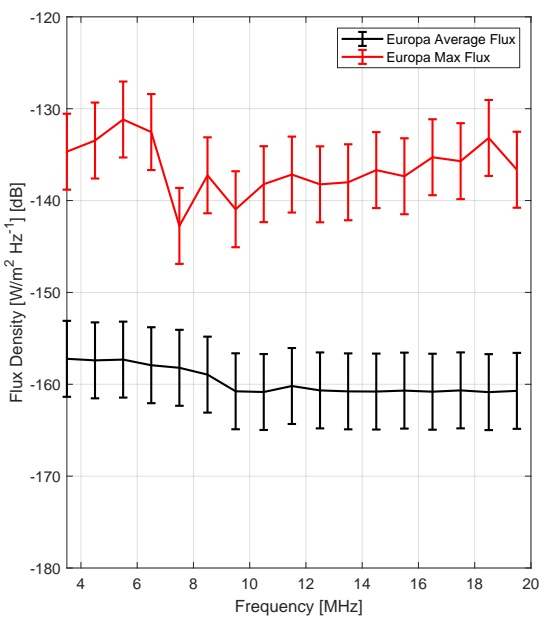

Fig. 11: Europa Jovian DAM flux density values (mean and max) versus emission frequency. The error bars point out the uncertainty on the JUNO Waves electrical antenna length assumed in the range $0.46 \mathrm{~m} \leq L_{\text {eff }} \leq 2.41 \mathrm{~m}$. The maximum and average power levels for Ganymede and Callisto can be derived by subtracting $4 \mathrm{~dB}$ and $9 \mathrm{~dB}$, respectively, from the provided curves.

Jovian DAM events with emission frequency smaller than 3.5 MHz or greater than 19.5 MHz are discarded (see Section III-A). Moreover, to compile statistics, we only focus on emissions that are above the galactic noise by at least 10 dB. In practical terms, we consider only emissions such that their normalized PSD over the sensitivity $P \bar{S} D(f, t)$ (see (21)) is greater than an effective threshold equal to $10-20 \log 10\left(2.41[m] / L_{\text {eff }}\right)-C(f)[\mathrm{dB}]$, where $C(f)$ is a frequency dependent correction factor (in the order of $1 \mathrm{~dB}$ ) evaluated at $L_{\text {eff }}=2.41 \mathrm{~m}$ that is equal to the $\mathrm{dB}$ difference between the value of the JUNO sensitivity $N_{b}(f)$ and of the galactic noise at each frequency (see Fig. 10).

The Jovian DAM occurrence probability $P_{o}\left(C M L, \varphi_{I o}, f\right)$ with respect to the $\mathrm{CML}$, Io phase $\varphi_{I o}$ and emission frequency $f$ is computed from the previously described Jovian DAM emissions catalogue as:

$$
P_{o}\left(C M L, \varphi_{I o}, f\right)=\frac{N_{o}\left(C M L, \varphi_{I o}, f\right)}{N_{t}\left(C M L, \varphi_{I o}, f\right)}
$$

where $N_{o}$ is the cumulative number of hours for which the Jupiter DAM is detected and $N_{t}$ the total number of hours listening for a given CML bin vs Io phase bin and emission frequency. The experimental data is organized in rectangular bins of size equal to $18^{\circ} \times 18^{\circ}$.

As first step, in order to understand how the spatial occurrence probability varies as function of $L_{\text {eff }}$, we performed a parametric monodimensional experiment by computing the probability of occurrence versus the Io phase $\varphi_{I o}$ (similar results holds for the CML case). This is propaedeutic for computing the bidimensional spatial occurrence as described in (22).

Without loss of generality, Fig. 12 reports the results of this analysis assuming $f=3.5 \mathrm{MHz}$ (similar results can be obtained at different emission frequencies). These results show a strong variation of the occurrence probability versus $L_{\text {eff }}$. Given that we assume $10 \mathrm{~dB}$ above galactic noise as threshold, the retrieved occurrence values for $L_{\text {eff }}<1 \mathrm{~m}$ and $L_{\text {eff }}>1.46$ appear unlikely as they are either too low or too high based on comparison with previous literature [31], [32], [33], [34]. This result is in line with what previously estimated in [29], [30]. The two observed spikes in spatial occurrence probability roughly centered at $\varphi_{I o}=75^{\circ}$ and $\varphi_{I o}=250^{\circ}$ are expected from the literature albeit typically observed at higher emission frequencies [32].

Without loss of generality, Fig. 13, Fig. 14 and 15 report the contour maps of $P_{o}\left(C M L, \varphi_{I o}, f\right)$ as function of three different values of $L_{\text {eff }}$ for selected frequencies of $3.5 \mathrm{MHz}$, $9.5 \mathrm{MHz}$ and $18.5 \mathrm{MHz}$ respectively. By assuming $L_{\mathrm{eff}}=1$, the probability of occurrence peaks at about 0.4 for $3.5 \mathrm{MHz}$ and $9.5 \mathrm{MHz}$ while it peaks at about 0.9 for $18.5 \mathrm{MHz}$. For higher values of $L_{\text {eff }}$, the probability of occurrence decrease. It is interesting to note that the high occurrence regions in the CML and Io phase plane at 3.5 MHz and 9.5 MHz, and in the lower $\mathrm{MHz}$ frequency range in general, are considerably different with respect to the typical Io controlled regions observed at 18.5 MHz from Earth based observations [31] (see Fig. 3) and by JUNO waves (see Fig. 15).

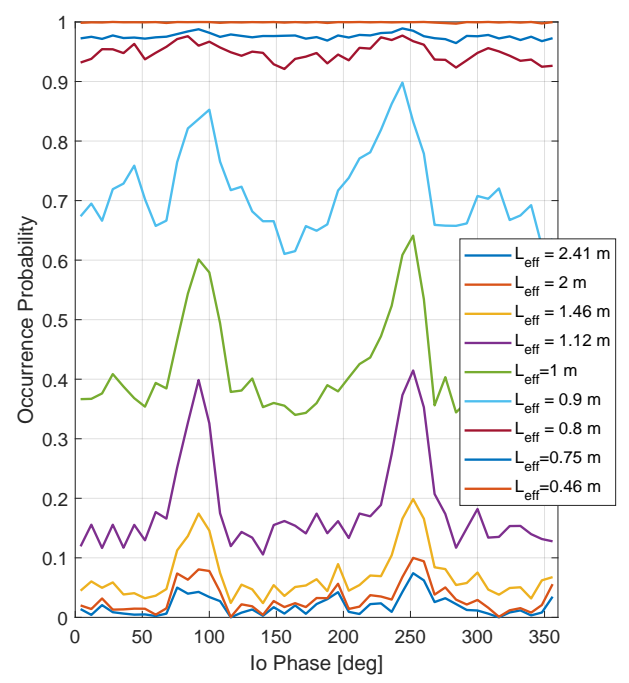

Fig. 12: Jovian DAM spatial occurrence probability versus the Io Phase $\varphi_{I o}$ as a function of $L_{\text {eff }}$ at $f=3.5 \mathrm{MHz}$.

Fig. 16 reports the daily occurrence of the Jovian DAM versus the emission frequency for 322 days of consecutive observations for three different values of $L_{\text {eff }}$ (see previous discussion). The daily occurrence is defined as the number of hours per day in which Jupiter emissions are detected at a given frequency. The results on daily occurrence show that Jovian DAM has an average probability occurrence of 0.2 , which corresponds to roughly 5 hours of cumulative observation time of Jovian DAM emissions over a day. Higher occurrence values are reported in the frequency range between 


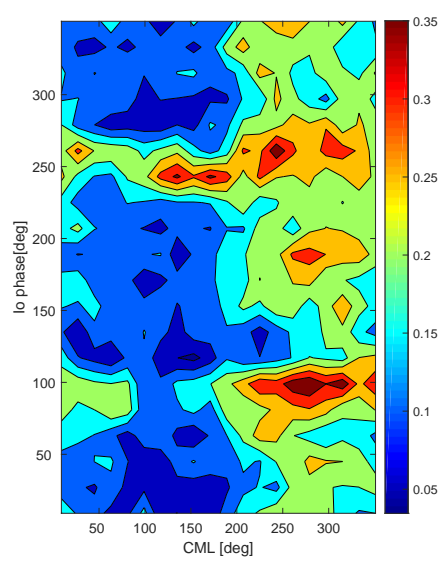

(a)

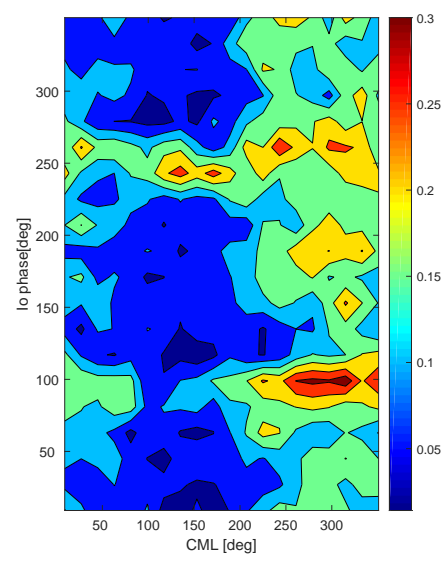

(b)

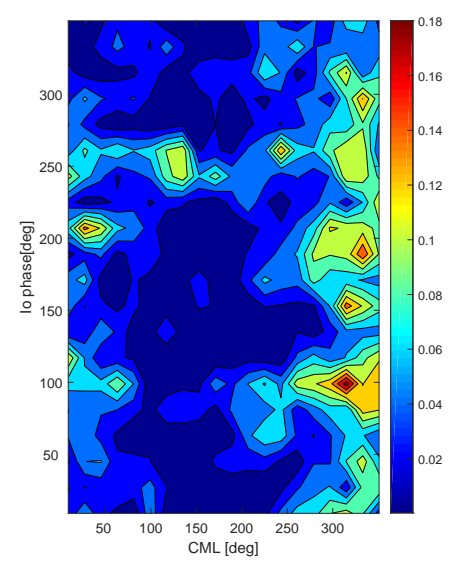

(c)

Fig. 13: Contour maps of the Jovian DAM occurrence probability $P_{o}\left(C M L, \varphi_{I o}, f\right)$ for $f=3.5 \mathrm{MHz}$ assuming (a) $L_{\text {eff }}=1 \mathrm{~m}$, (b) $L_{\text {eff }}=1.46 \mathrm{~m}$ and (c) $L_{\text {eff }}=2.41 \mathrm{~m}$.

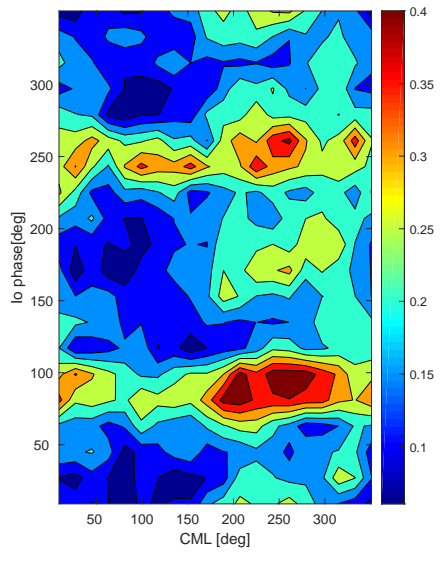

(a)

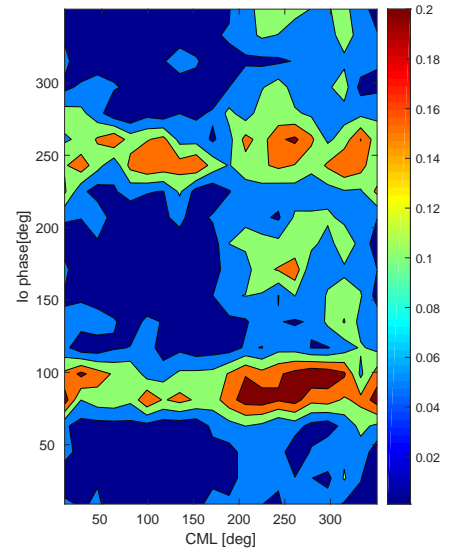

(b)

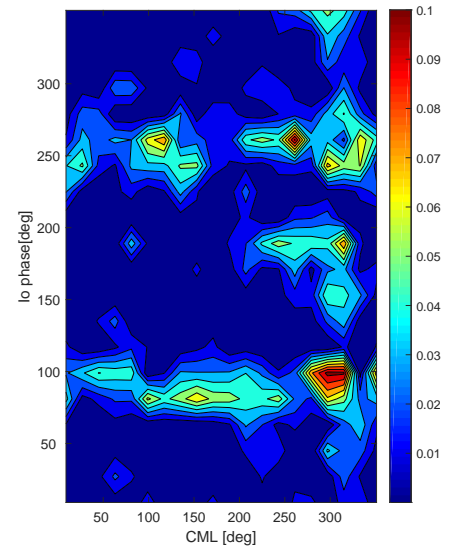

(c)

Fig. 14: Contour maps of the Jovian DAM occurrence probability $P_{o}\left(C M L, \varphi_{I o}, f\right)$ for $f=9.5 \mathrm{MHz}$ assuming (a) $L_{\text {eff }}=1 \mathrm{~m}$, (b) $L_{\text {eff }}=1.46 \mathrm{~m}$ and (c) $L_{\text {eff }}=2.41 \mathrm{~m}$.

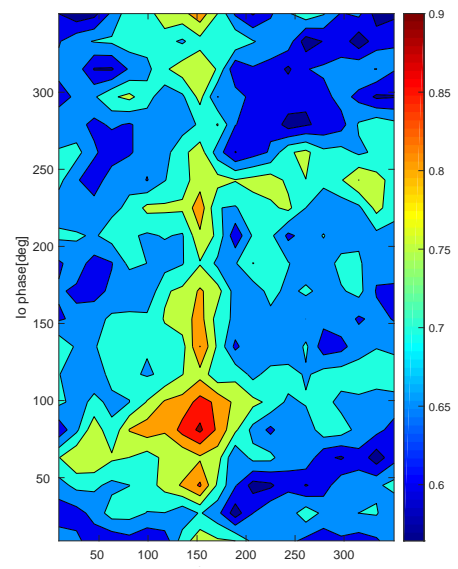

(a)

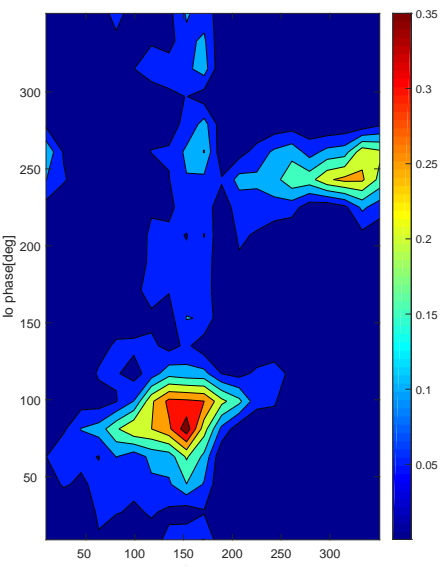

(b)

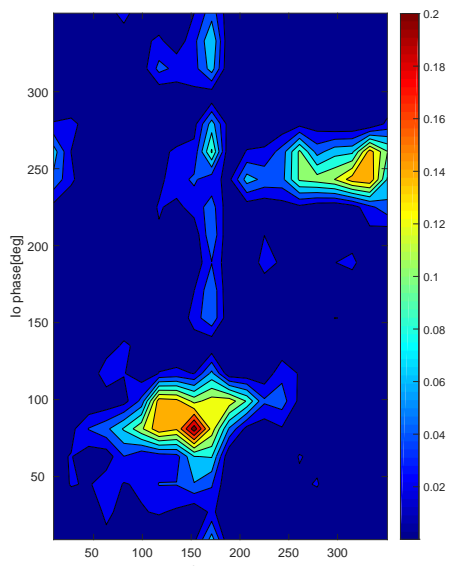

(c)

Fig. 15: Contour maps of the Jovian DAM occurrence probability $P_{o}\left(C M L, \varphi_{I o}, f\right)$ for $f=18.5 \mathrm{MHz}$ assuming (a) $L_{\mathrm{eff}}=1 \mathrm{~m}$, (b) $L_{\text {eff }}=1.46 \mathrm{~m}$ and (c) $L_{\text {eff }}=2.41 \mathrm{~m}$. 
16.5 $\mathrm{MHz}$ and $18.5 \mathrm{MHz}$ for $L_{\mathrm{eff}}=1 \mathrm{~m}$.

These results are useful for both planning passive radar acquisition and defining the passive radar setup (e.g., working frequency and bandwidth). Moreover, they can be exploited for planning active and passive radar acquisitions. To this extent, the computation of the occurrence probability $P_{o}\left(C M L, \varphi_{I o}, f\right)$ allows to quantify the likelihood of encountering Jovian DAM for a given observer (e.g., passive probe) location and given acquisition time window. The differential CML (denoted $\triangle C M L$ ) and Io phase (denoted as $\Delta \varphi_{I o}$ ) as function of the overall acquisition time $t$ for an observer on a given icy moon is computed as (see Fig. 3):

$$
\begin{gathered}
\Delta C M L(t)=\left(\omega_{j}-\omega\right) t \\
\Delta \varphi_{I o}(t)=\left(\omega_{i o}-\omega\right) t
\end{gathered}
$$

where $\omega_{j}, \omega_{i o}$ and $\omega[\mathrm{deg} / \mathrm{s}]$ are the angular velocity of Jupiter (sidereal), Io and the target icy moon (i.e., Europa, Ganymede or Callisto) respectively.

The occurrence probability over a random statistical trajectory is computed by first dividing the overall acquisition time $t$ in non-overlapping segments of duration $\Delta t$ denoted as acquisition time and then by selecting the maximum value of $P_{o}\left(\Delta C M L(t), \Delta \varphi_{I o}(t), f\right)$ along each trajectory segment.

The overall statistics for the random trajectory assessment (mean and absolute deviation) are provided in Fig. 17 for different values of $L_{\text {eff }}$ and different emission frequencies across the considered range. These statistics are provided as function of a parametric acquisition time 5 minutes $\leq \Delta t \leq$ 3 hours. The results show that, across the emission frequency range, the probability of encountering Jovian DAM is similar and increases with the acquisition time with a maximum value of about 0.4. An exception (not shown) is given by emission frequencies around $18.5 \mathrm{MHz}$ that exhibit an average probability that peaks at about 0.7 for $L_{\text {eff }}=1 \mathrm{~m}$. This can also be seen from Fig. 15 (a). Notably, the Europa, Ganymede and Callisto cases hold very similar results. Obviously, if we consider the entire range between $3.5 \mathrm{MHz}$ and $19.5 \mathrm{MHz}$, the probability of occurrence is likely to increase. Accordingly, a wide band receiver should be the preferred implementation for passive sounding. Moreover, this analysis also suggests that an orbiting or lander configuration with long passive observation ( $\Delta t$ in the order of many hours) is preferred to a flyby one in order to maximize the probability of encountering Jovian DAM radiation. These long observations may have an impact on the data volume which can be reduced by an additional on-board processing strategies.

\section{Jovian Noise Time-Bandwidth Product}

In this section we investigate the mean achievable integration time and bandwidth of Jovian DAM and derive the mean achievable time-bandwidth product $\Omega$ (see Section II). The provided values should be interpreted as the maximum continuous useful burst bandwidth and integration time thus they could be subdivided into smaller subsets. We consider as a potential passive sounding signal a Jupiter emission such that its power spectral density $P \bar{S} D(f, t)$ (i) remains approximately constant across a certain bandwidth $B_{e}$ and

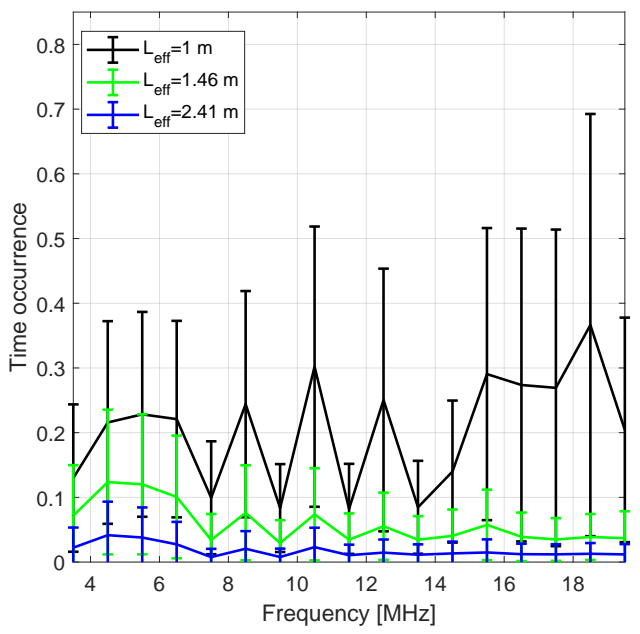

Fig. 16: Jovian noise temporal occurrence (mean and standard deviation) over a day versus the emission frequency and as function of $L_{\text {eff. }}$.

integration time $T_{e}$ and, as in the previous section, (ii) is above the galactic noise by at least $10 \mathrm{~dB}$ (as in previous section). Fig. 18 reports an example illustrating where useful Jovian DAM emission have been detected and characterized according to our procedure as a function of $B_{e}, T_{e}$ and $\Omega$. As in previous sections, we first investigate the statistical variations of the retrieved parameters as function of $L_{\text {eff }}$. Fig. 19 reports the results of this analysis. The same considerations on $L_{\mathrm{eff}}$ reported in Section III-C apply to this analysis. Accordingly, we estimate the average useful burst bandwidth $B_{e}=2.5 \pm 0.5$ $\mathrm{MHz}$ and the average integration time $T_{e}=8 \pm 1.8$ minutes by assuming $1 \leq L_{\text {eff }} \leq 1.46$. Thus, in this case, the actual value of the effective antenna length is not considerably changing the results.

The estimated values of $B_{e}$ and $T_{e}$ provide an average time-bandwidth product $2 \Omega=93 \mathrm{~dB}$. However, as stated in Section II, the integration time is limited by the $\mathrm{S} / \mathrm{C}$ motion to the order of seconds. Therefore this value of time-bandwidth product can be reached only with a lander. To illustrate the situation with an integration time compatible with a spacecraft acquisition, without loss of generality, Fig. 20 reports the results on the burst bandwidth distribution assuming $T_{e}=1$ $\mathrm{s}$ and $L_{\mathrm{eff}}=1 \mathrm{~m}$. In this case, The average burst bandwidth is about $3 \mathrm{MHz}$ resulting in an average $\Omega=65 \mathrm{~dB}$. Fig. 20 (b) reports the maximum allowable value of the two-way subsurface losses $L_{s s}$ (see (14)) as a function of the emission frequency and slope assuming $h=500 \mathrm{~km}$ for $\Omega=65 \mathrm{~dB}$. For moderate characteristic slopes values, the maximum allowable value of $L_{s s}$ such that it is possible to detect a subsurface reflector ranges from 50 to $65 \mathrm{~dB}$ which is within the expected range considered in [25].

\section{E. Jovian Noise Spectral Structure Fitness for Passive Sound- ing}

We analyze JUNO Burst data products described in Section III-A to evaluate the Jovian noise spectral flatness and its 


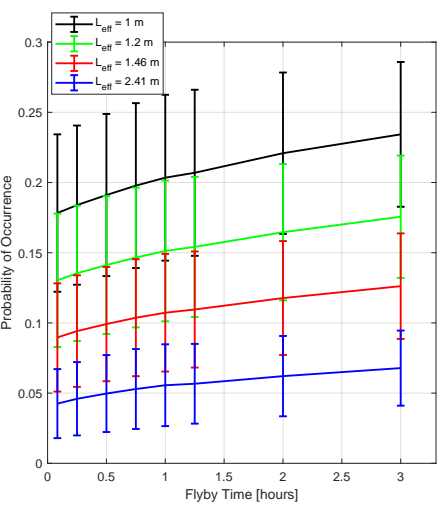

(a)

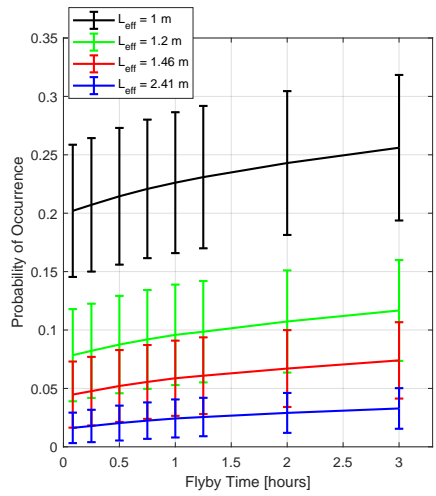

(b)

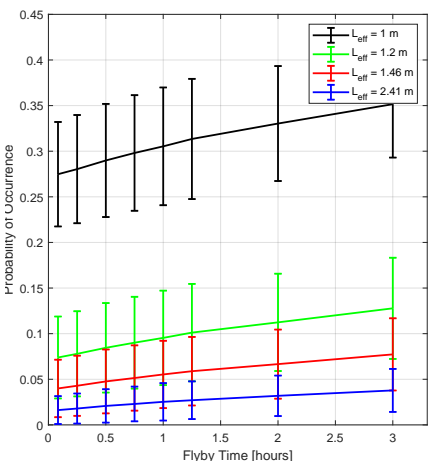

(c)

Fig. 17: Overall random trajectories statistics for the probability of Jovian DAM occurrence for a variable acquisition time $\Delta t$ and different values of $L_{\text {eff. }}$ (a) Results for $f=3.5 \mathrm{MHz}$, (b) $f=9.5 \mathrm{MHz}$ and (c) $f=14.5 \mathrm{MHz}$. Statistics were computed from segmenting, according to $\Delta t$, a time frame $t$ equal to 400 days (i.e. $0 \leq t \leq 400$ days). Europa, Callisto and Ganymede cases hold similar results.

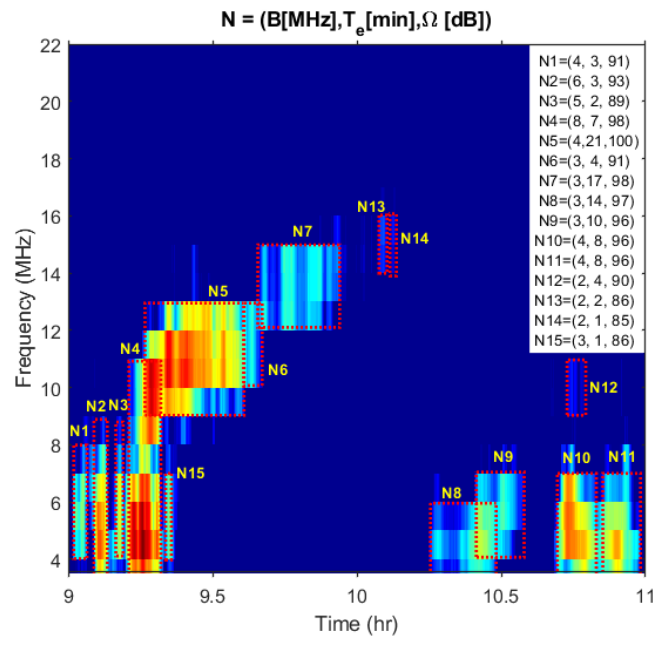

Fig. 18: Example of detected bursts for SURVEY data product (2017-129) from 09:00 to 11:00. For each burst, the bandwidth $B$, the integration time $T_{e}$ and time-bandwidth product $\Omega$ is reported.

autocorrelation properties. Then we compared it with theoretical white noise with similar bandwidth and integration time. This dataset spectrograms are characterized by waveforms of bandwidth $B=1.31 \mathrm{MHz}$ and integration time $T_{s}=3.12$ ms. In the gaussian white band-limited noise hypothesis case, this corresponds to a constant sidelobe level equal to $2 \Omega=39$ $\mathrm{dB}$ and a range resolution proportional to $1 / B=0.76 \mu \mathrm{s}$. The burst spectrogram durations are variable (i.e., number of stacked waveforms) and are in the order of minutes. The overall list of experiments is shown in Table I. At the current time, this represents the totality of high-resolution spectrograms available from JUNO data. Data has been preprocessed by notching narrow band radio frequency interference spikes randomly appearing in the power spectral density. These spectral spikes originated from the JUNO spacecraft and electronics. The normalized autocorrelation functions are computed from experimental power spectral densities accord-

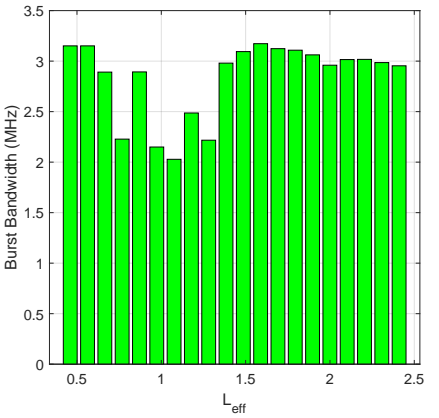

(a)

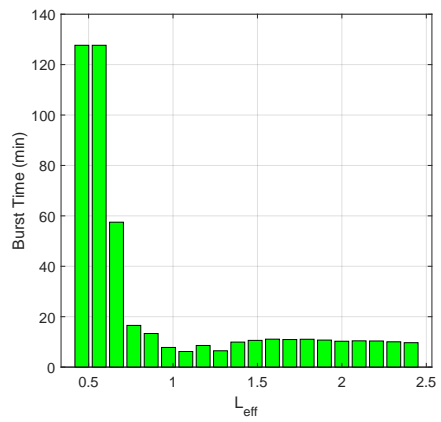

(b)

Fig. 19: Estimated values of the (a) useful bandwidth $B_{e}$ and (b) integration time $T_{e}$ versus $L_{\text {eff. }}$ The relevant integration time for a spacecraft in motion is in the order of 1 second.

ing to (4). The experimental data (see examples of Fig. 21 (c) and 22 (c)) show that the Jovian DAM power spectral density can be modelled as in (16), where $S(f)$ is the power spectral density of band-limited white gaussian noise (i.e. fast variations in the PSD) and $K(f)$ models the slowly-varying correlated variations. The results show that for small time lags (i.e., autocorrelation main lobe region), the slow correlated variations dominate the response and introduce a substantial 


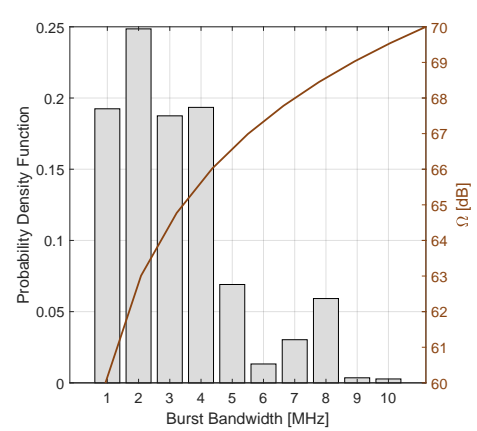

(a)

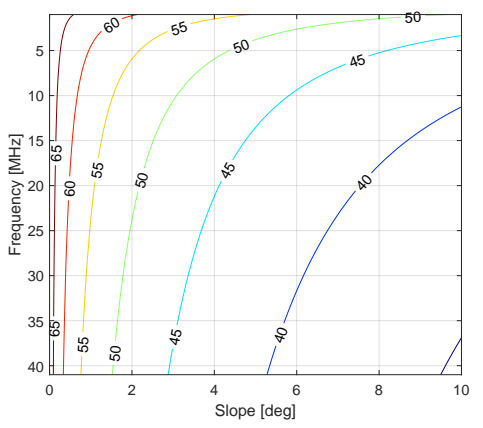

(b)

Fig. 20: (a) Example of probability density function of the Jovian DAM useful bandwidth $B_{e}$ assuming $T_{e}=1 \mathrm{~s}$ and $L_{\mathrm{eff}}=1 \mathrm{~m}$. The value of $\Omega$ is also reported. (b) Contour plot of the maximum allowable two-way subsurface losses (i.e. loss budget with no specific assumption on subsurface scenario) $L_{s s}[\mathrm{~dB}]$ (see (14)) as function of the emission frequency and surface slope $\sigma_{s}$. The plot assumes $B_{e}=3 \mathrm{MHz}$ and $T_{e}=1 \mathrm{~s}$ resulting in $\Omega=65 \mathrm{~dB}$. Spacecraft height is assumed equal to $h=500 \mathrm{~km}$. $B_{e}=3 \mathrm{MHz}$ is the average value of the distribution reported in the upper panel.

discrepancy between the assumed theoretical value of the autocorrelation function and the experimental one. The average resolution is a factor 3 wider compared to the white noise case (see Table I). For large delays, the Jovian noise autocorrelation function approaches the theoretical white noise floor with an average discrepancy of $5 \mathrm{~dB}$, which is a small increase in the noise floor.

Nevertheless, it is possible to improve the autocorrelation sidelobes by mitigating the effect of $K(f)$ by simple power trimming procedure. This is performed by first estimating the statistical distribution of the values of $S(f)$ by selecting a spectral region where $K(f)$ is negligible, if exists, such as the 4.4 to $4.45 \mathrm{MHz}$ spectral region of Fig. 21c. After the estimation procedure is performed, samples that exceed the maximum or fall under the minimum of the previously estimated distribution are notched from the overall power spectral density $\tilde{S}(f)$. Then the removed samples are replaced with estimates extrapolated from forward and reverse autoregressive fits of the remaining samples [35]. Fig. 23 shows an example of results of the described procedure. Noise floor level improvement is about $8 \mathrm{~dB}$ across the entire waveform. This power equalization procedure will affect the power contained in $\tilde{S}(f)$, depending on the values of the coefficient $a_{n}$ of $K(f)$, and in turns the echo peak power. In this example the power loss is about $8+/-4 \mathrm{~dB}$. There is the possibility of scaling the approach performance by reducing the threshold on the power trimming after the estimation procedure of $S(f)$. Therefore there is a variable trade-off between power loss and amount of noise floor reduction. More sophisticated techniques that fit $K(f)$ to the spectrum and then apply the correction might perform better than our proposed approach. This will be subject of a future study.

\section{F. Implications on RIME and REASON Passive Performance and Operations}

In the following, we evaluate the general results reported in Section III-B to Section III-D in the perspective of the planned radar sounding mission to the Jupiter's Moons, namely RIME and REASON. Both RIME and REASON HF antennas are operating at $9 \mathrm{MHz}$ central frequency.

Table II reports the derived average and maximum Jovian DAM flux density for the different moons at $9.5 \mathrm{MHz}$. The standard deviation of the measurement, that is due to the uncertainty on the value of the $L_{\text {eff }}$ (assumed to be in the range 0.46 to $2.41 \mathrm{~m}$ ), is equal to $\pm 4 \mathrm{~dB}$. The galactic noise level flux density level at $9.5 \mathrm{MHz}$ is in the order of $10^{-19}$ $\left[\mathrm{Wm}^{-2} \mathrm{~Hz}^{-1}\right]$ (see Fig. 2). By assuming the values reported in Table II, we derived the Jovian DAM mean relative power level with respect to it. The mean value of this ratio is between 23 and $31 \mathrm{~dB}$ depending on the icy moon.

The analyses on time-bandwidth product and temporal occurrence are done by exploiting the available orbital information [36] for the planned flybys of RIME and REASON on Europa and for RIME flybys on Ganymede and Callisto. The exploited kernels are CREMA 3.2 for RIME and 13-F7 for REASON. In the RIME, case the evaluation is performed also for the orbital phase around Ganymede.

By taking into account the expected spacecraft flyby velocities and radar parameters, the estimated average value of the time-bandwidth product $2 \Omega$ [see (6),(7)] is equal to $70.5+/-$ $1.5 \mathrm{~dB}$ for RIME and equal to $66+/-2.4 \mathrm{~dB}$ for REASON. In the RIME case, the provided value of $\Omega$ is representative for all the different flybys on Europa, Ganymede and Callisto. At the closest approach, which represents the worst case scenario, the value of $2 \Omega$ is equal to $68 \mathrm{~dB}$ for $\operatorname{RIME}(h=200 \mathrm{~km})$ and equal to $58.5 \mathrm{~dB}$ for the REASON case $(h=20 \mathrm{~km})$. The RIME orbital phase around Ganymede $(h=500 \mathrm{~km})$ results in $2 \Omega=76 \mathrm{~dB}$.

Fig. 24 reports the values of the maximum two-way allowable subsurface losses $L_{s s}$ (see (14)) versus the characteristic surface slope $\sigma_{s}$ in the worst case scenario for RIME and REASON (i.e., closest approach). For moderate characteristic slopes values, the worst case value of $L_{s s}$ is deemed acceptable for an actual subsurface interface detection. The subsurface losses from the icy moons are expected to be in the range between 50 and $65 \mathrm{~dB}$ [6], [25].

To evaluate the probability of spatial occurrence, we exploit the derived value of $P_{o}\left[C M L(t), \varphi_{I o}(t), f\right]$ at $9.5 \mathrm{MHz}$ (see Fig. 14) as in (22). Then, based on these occurrence maps 


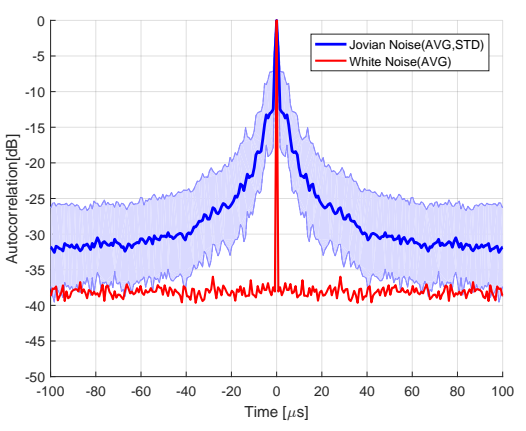

(a)

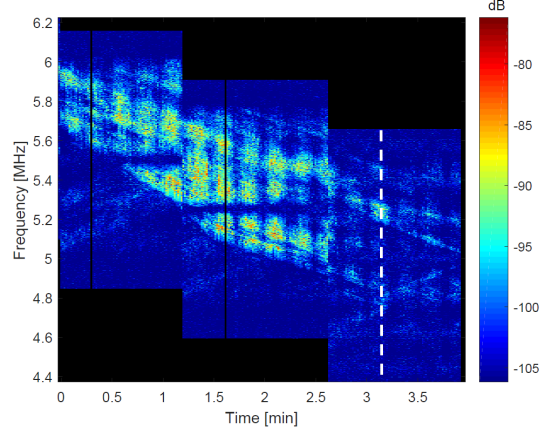

(b)

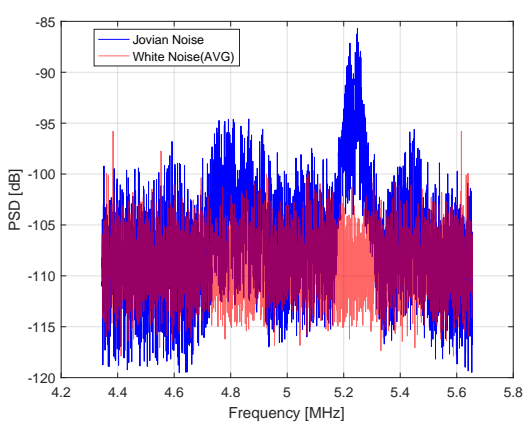

(c)

Fig. 21: (a) Normalized autocorrelation structure comparison between Jovian DAM emission (average and standard deviation) and theoretical white noise (average). The bandwidth is equal to $B=1.31 \mathrm{MHz}$ and integration time $T_{s}=3.12 \mathrm{~ms}$. (b) Jovian noise burst spectrogram acquired by JUNO on Orbit 01, 2016-08-27 (240) used to computed the Jovian noise autocorrelations. (c) Example of a realization of Jovian noise Power Spectral Density versus frequency (i.e. white dashed line in Figure (b)) compared to theoretical white noise.

TABLE I: JUNO Waves data used for evaluating the Spectral Structure of Jovian Noise in terms of Autocorrelation and resulting performance with respect to theoretical band-limited gaussian noise with same bandwidth and integration time.

\begin{tabular}{|c||c||c||c||c|}
\hline JUNO Waves Burst Data ID & Burst Duration [min] & Emission Frequency [MHz] & Noise Floor Increase[dB] & Resolution Broadening Factor \\
\hline $2016-240$ & 3.7 & 5.3 & 6 & 2 \\
\hline $2016-346$ & 6 & 5 & 7 & 3 \\
\hline $2016-346$ & 5.5 & 6.5 & 3 & 2 \\
\hline $2017-033$ & 3 & 7.5 & 3 & 3 \\
\hline $2017-033$ & 6 & 5 & 3 & 2 \\
\hline $2017-086$ & 0.6 & 21 & 6 & 6 \\
\hline $2017-086$ & 2 & 3.8 & 4 & 2 \\
\hline $2017-139$ & 2 & 3.8 & 3 & 6 \\
\hline
\end{tabular}

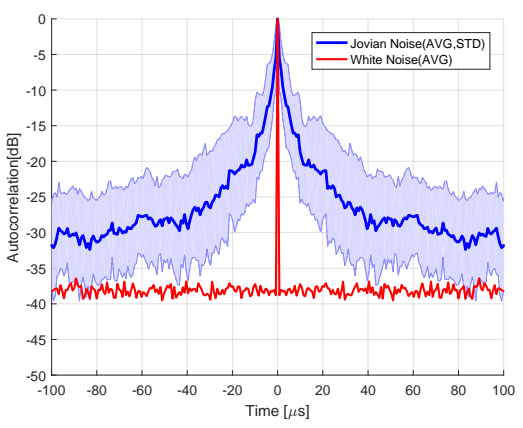

(a)

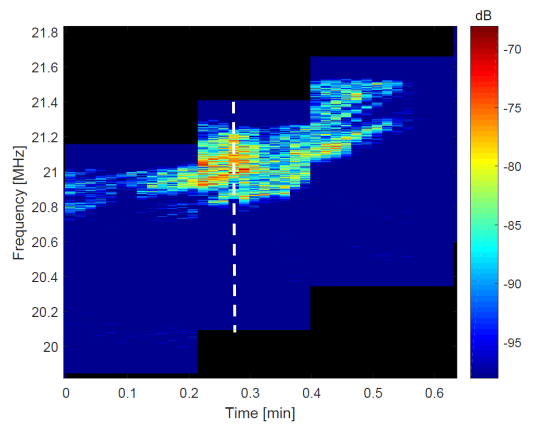

(b)

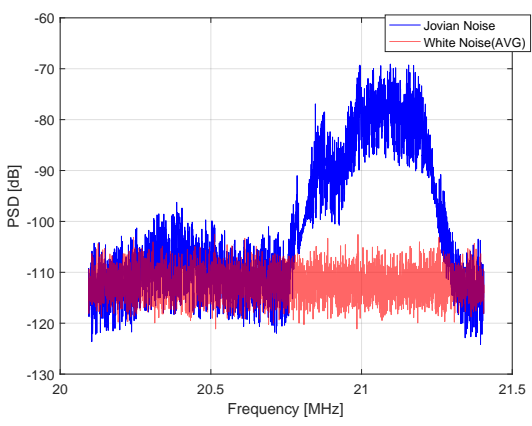

(c)

Fig. 22: (a) Autocorrelation structure comparison between Jovian DAM emission (average and standard deviation) and theoretical white noise (average). The bandwidth is equal to $B=1.31 \mathrm{MHz}$ and integration time $T_{s}=3.12 \mathrm{~ms}$. (b) Jovian noise burst spectrogram acquired by JUNO on Orbit 05, 2017-03-27 (086) used to computed the Jovian noise autocorrelations. (c) Example of a realization of Jovian noise Power Spectral Density versus frequency (i.e. white dashed line in Figure (b)) compared to theoretical white noise.

that are function of $L_{\text {eff }}$, we performed a trajectory analysis by extrapolating the actual value of $C M L(t)$ and $\varphi_{I o}(t)$ from the orbital kernels.

In the flyby cases, the results of this analysis (see Fig. 25 and Fig. 26) show that the probability of occurrence is rather small and flyby dependent for both Europa, Ganymede and Callisto cases. The maximum probability of occurrence of Jovian DAM is about 0.3 for selected REASON flybys on Europa.

According to orbital kernels, the RIME circular orbit phase around Ganymede is planned from 2033-046 to 2033-176
(ISO 8601 ordinal date) resulting in 131 days of observations. During the orbital phase of mission, the available observing time is much greater than during flybys, so data volumes are too great to consider recording continuously. Therefore, we identify potential passive opportunities based on the value of the probability of occurrence $P_{o}\left(C M L(t), \varphi_{I o}(t), f\right)$.

We consider as a potential passive acquisition opportunity when the spacecraft is in a CML/IO phase bin with occurrence probability greater than one standard deviation (i.e. high occurrence probability bins) with respect to the mean of the overall distribution of $P_{o}\left(C M L, \varphi_{I o}, f\right)$ at $f=9.5 \mathrm{MHz}$. 


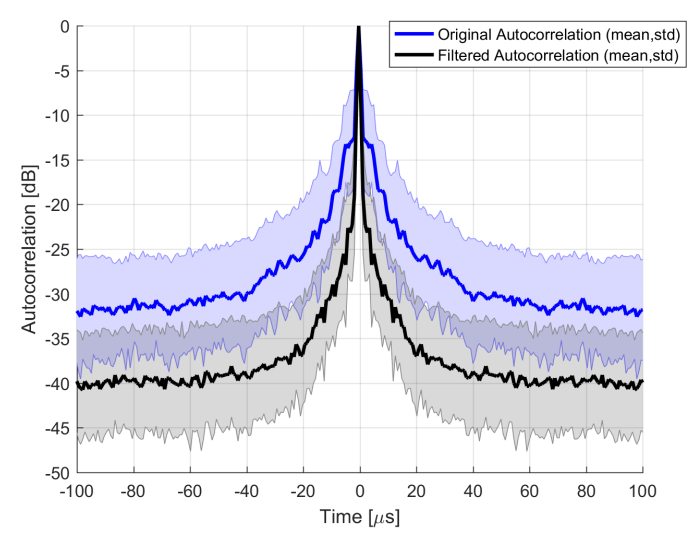

Fig. 23: Example of normalized Autocorrelation improvement by thresholding and autoregression filtering on experimental results of Fig 21.

Fig. 27 qualitatively reports the results of this analysis by showing the identified tracks over the occurrence contour map at $9.5 \mathrm{MHz}$ and $L_{\text {eff }}=1 \mathrm{~m}$ (see Fig. 14 (a)). Based on our assumptions, Table III reports the quantitative results on the duration of the time window and the number of opportunities as function of $L_{\text {eff }}$.

We can expect an average persistence of the spacecraft on high emission region in the order of 45 minutes (see Table III) and a number of opportunities between 500 and 600. Knowing that the RIME Ganymede circular orbit phase lasts 131 days, this results in roughly four passive acquisition opportunities every day that is a notable result.

TABLE II: Jovian DAM Flux Density statistics measured in the proximity of the Moons at $9.5 \mathrm{MHz}$. Values are reported considering the uncertainty on the JUNO Waves electric antenna legth $L_{\text {eff }}$ (see Section III-A).

\begin{tabular}{c||c||c||c|} 
& Europa & Ganymede & Callisto \\
\hline Mean Flux Density dB $\left[\mathrm{Wm}^{-2} \mathrm{~Hz}^{-1}\right]$ & $-159 \pm 4$ & $-163 \pm 4$ & $-167 \pm 4$ \\
\hline Max Flux Density dB $\left[\mathrm{Wm}^{-2} \mathrm{~Hz}^{-1}\right]$ & $-137 \pm 4$ & $-141 \pm 4$ & $-145 \pm 4$ \\
\hline Mean relative Power [dB] over galactic noise & $31 \pm 4$ & $27 \pm 4$ & $23 \pm 4$ \\
\hline
\end{tabular}

\section{DISCUSSION}

The understanding of the in-situ temporal and spectral properties of the Jovian Decametric Radiation (DAM) is of fundamental importance for a more realistic assessment of the performance of radar passive sounding acquisition of Jupiter's moon subsurface and for planning active sub-jovian observations in HF band.

The uncertainty on the JUNO Waves effective electric antenna length $L_{\text {eff }}$ poses a limit to the presented analyses

TABLE III: Summary of results on the distribution of time duration (i.e. persistence on high emission regions) of identified passive acquisition opportunities for RIME circular orbit phase around Ganymede as function of $L_{\text {eff }}$.

\begin{tabular}{c||c||c}
$L_{\text {eff }}[\mathrm{m}]$ & Opportunity time [min] & Number of Opportunities \\
\hline 1 & $51 \pm 31$ & 448 \\
\hline 1.46 & $43 \pm 21$ & 626 \\
\hline 2.41 & $39 \pm 20$ & 701 \\
\hline
\end{tabular}

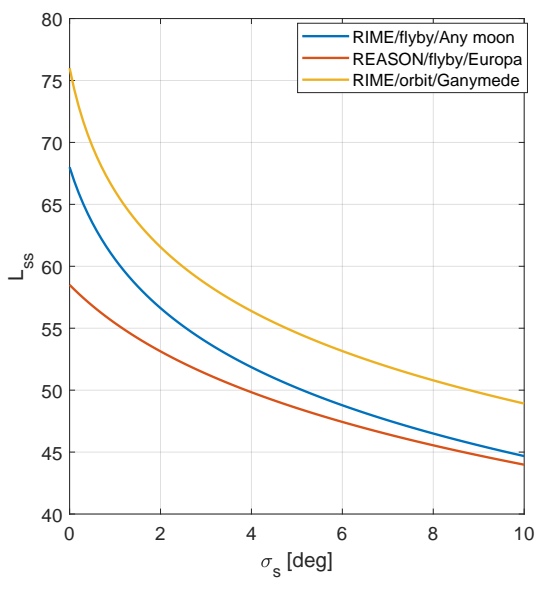

Fig. 24: Worst case value of the maximum allowable two-way subsurface losses $L_{s s}$ versus the characteristic slope $\sigma_{s}$ for RIME and REASON (flyby and orbit).

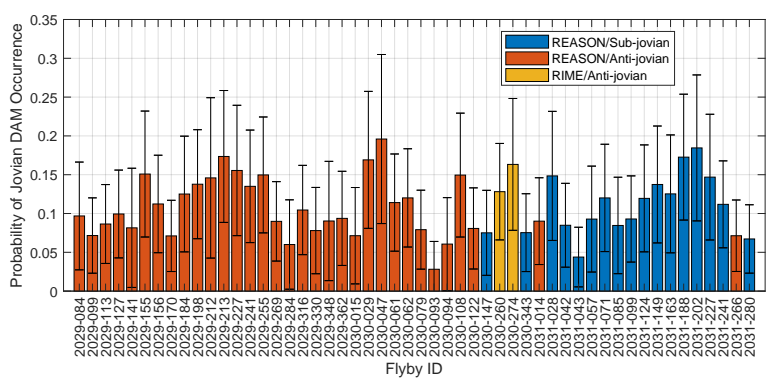

Fig. 25: Probability of Jovian DAM occurrence for REASON (red and blue) and RIME (yellow) planned flybys on Europa. Flyby ID is year - day of the year (ISO 8601 ordinal date). The error bars represent the measurement standard deviation resulting from the uncertainty on the actual value of the JUNO Waves electrical antenna $L_{\text {eff }}$ assumed to be in the range between $1 \mathrm{~m}$ and $2.41 \mathrm{~m}$.

(see Section III-A). As discussed, previous literature bounds its value in the range $0.46 \leq L_{\text {eff }} \leq 2.41$. The parametric analyses performed in Section III indicate that the actual value of $L_{\text {eff }}$ is likely to be in the range between $1 \mathrm{~m}$ and $1.46 \mathrm{~m}$. This is in line with what reported in [22], [29]. Assuming a $10 \mathrm{~dB}$ threshold over galactic noise for Jovian DAM emissions evaluation, all the derived metrics, such as spatial and temporal occurrence, improve for shorter antenna lengths with respect to longer ones. On the one hand, the presented results on spatial occurrence value are moderately sensitive to variations in $L_{\text {eff }}$ values. On the other hand, the location of high emission regions across the frequency range under investigation is almost invariant with $L_{\text {eff }}$ (e.g, Fig. 15). As a general remark, we can state that the derived metrics variations as function of $L_{\text {eff }}$ are moderate in the specific cases of RIME and REASON HF frequency band (both centered at 9 $\mathrm{MHz}$ ) while larger fluctuations are present in other frequency bands. As an example, when the portion of the spectrum at 18.5 $\mathrm{MHz}$ is considered, the occurrence of emission range 


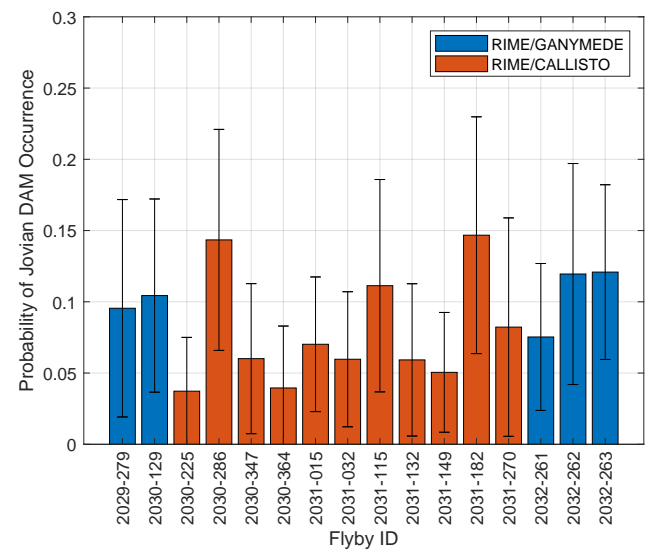

Fig. 26: Probability of Jovian DAM occurrence for RIME flybys on Ganymede (red) and Callisto (blue). Flyby ID is year - day of the year (ISO 8601 ordinal date). All the flybys are predominantly antijovian. The error bars represent the measurement standard deviation resulting from the uncertainty on the actual value of the JUNO Waves electrical antenna $L_{\text {eff }}$ assumed to be in the range between $1 \mathrm{~m}$ and $2.41 \mathrm{~m}$.

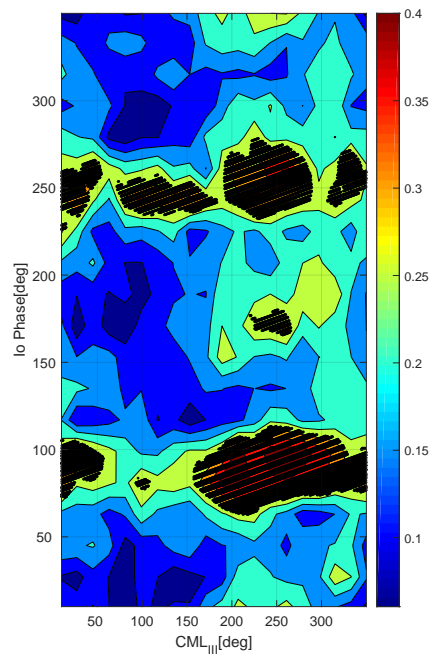

Fig. 27: Overlay between the identified passive acquisition opportunities spacecraft tracks for the RIME circular orbit phase around Ganymede and the probability of occurrence $P_{o}\left(C M L, \varphi_{I o}, f\right)$ contour map (assumption of $L_{\text {eff }}=1 \mathrm{~m}$.)

from very rare to nearly ubiquitous depending on the value assumed for $L_{\text {eff. }}$.

In terms of time-bandwidth product, the performed analyses of Jovian DAM show that there is a reasonable margin for subsurface interface detection from orbit even if the actual thermal and structural conditions of the icy moon's subsurface can only be hypothesized. This also applies when the active RIME and REASON orbital and operational parameters are considered.

Until now, operations for the RIME and REASON instruments at $9 \mathrm{MHz}$ (HF band) have been planned by assuming acquisitions on the anti-jovian side of the icy moons. This is required for mitigating the risk of Jovian emission interfering with the active radar echoes reception. The results of this paper on sporadic temporal occurrence of the Jovian DAM at $9.5 \mathrm{MHz}$ (see Section III-F) could potentially open up sub-jovian acquisitions by the upcoming REASON and RIME instruments.

In the light of the analyses performed in this paper, some guidelines on passive sounding for planned and future missions can be provided. While it is not possible to extended RIME and REASON frequency range to accommodate a larger bandwidth, it is suggested to store in the on-board memory of the spacecraft an entire passive recording window of data (i.e. use the maximum possible sampling window length) and then downlink it. For future missions it would be strongly advisable to have on-board radio frequency interference filtering, spectral stitching, and auto-correlation processing (to reduce the data volume) capabilities. These observations were also recently reported by Peters et al. [37] for terrestrial experiments of passive sounding by exploiting the solar radiation. In particular, radio frequency interferences can deteriorate performance as they result an increase in the noise floor which could potentially mask subsurface reflections. Spectral stitching is a viable strategy for extending the passive sounding frequency range by merging the passive echoes acquired at non overlapping spectral sub-bandwidths.

\section{CONCLUSions}

In this paper, after introducing the principles of subsurface echo detection and waveform design for passive radar sounders, we investigated the spectral, spatial and temporal properties of the Jovian DAM for understanding its suitability for passive radar sounding of the Jupiter icy moons. This has been performed by taking advantage of the recently available Jovian DAM data acquired by JUNO Waves which is currently acquiring data around Jupiter.

The theoretical analysis shows that (i) the spectral structure of the Jovian DAM and (ii) the time-bandwidth product play fundamental roles in terms of passive radar subsurface echoes detection capability. Any deviation from the white noise assumption of the Jovian signal results in increased subsurface echo masking and degradation of the passive radar resolution. However, this can be mitigated or fully corrected. Deviation from white noise assumption, can also be caused by narrowband radio frequency interference generated by the passive instrument electronics. Therefore, it is of crucial importance to design a passive radar with very low distortions in the receiver chain as in the active radar case.

To reach an adequate value of time-bandwidth product for subsurface echo detection, the integration time should be in the order of seconds as demonstrated by the theoretical analysis. The signal bandwidth plays a marginal role when compared to the integration time, that is limited by the spacecraft motion.

The analysis of the JUNO Waves high spectral resolution data (i.e. burst data) shows that the Jovian DAM emissions spectral structure is not perfectly white. Performance degradation in terms of resolution and sensitivity loss are induced by slow-varying components in the signal power spectral density as predicted in the theoretical analysis. The average resolution 
degradation is a factor 3 and the noise floor increase is about $5 \mathrm{~dB}$ with respect to theoretical white noise. However, we demonstrated that this degradation can be compensated by simple additional processing to filter out the unwanted spectral components at the expense of peak power losses. We plan to develop more sophisticated approaches with improved performance in the future.

It is important to notice that the burst data considered in the analysis of Section III-E (see also Table I) have been acquired very close to Jupiter (about one Jovian radii) and at a high latitudes. The jovian DAM spectral content may depend on the latitude of the observer [38]. However, we could not find any low-latitude burst in the Juno data-set. Therefore our analyses on high resolution data have some uncertainty due to the latitudinal effect and on possible other signal propagation effects occurring between Jupiter and the target icy moon.

In the light of the analyses performed on the JUNO Survey data by also taking into account the uncertainty on $L_{\text {eff }}$ (see also Section IV), we can state the following.

The experimental analyses showed that the average useful Jovian DAM bandwidth is equal to $2.5 \pm 0.5 \mathrm{MHz}$ and the average integration time is equal to $8 \pm 1.8$ minutes. This results in an average time-bandwidth product of $93 \mathrm{~dB}$. However, this value can be reached only with a lander.

When the spacecraft case is considered, the average value of the time-bandwidth product $\Omega$ is equal to $65 \mathrm{~dB}$. This value has been computed by assuming 1 second of integration time. In both cases, the retrieved value of the time-bandwidth is satisfactory in terms of expected radar performance (see Section III-D).

To understand the spatio-temporal structure of the Jovian emission, we computed the DAM probability of occurrence as function of the CML vs Io Phase plane and the daily occurrence variability by exploiting about a year of JUNO observations. This is required to help defining the best trajectories for both passive and active mode operations and to understand the likelihood of encountering a Jovian emission. The temporal occurrence analysis shows that the Jovian DAM is more likely to occur for certain observation geometries and emission frequencies.

We exploited the computation of the probability of occurrence $P_{o}\left(C M L, \varphi_{I o}, f\right)$ to assess the probability of encountering Jovian DAM along a given flyby path with a limited time duration. In particular, the deterministic analysis performed by considering the actual orbital information for RIME and REASON and by assuming $1 \leq L_{\text {eff }} \leq 2.41 \mathrm{~m}$, shows that the probability of encountering a potential Jovian DAM emission at $9.5 \mathrm{MHz}$ is relatively low. The deployment of a wide band passive receiver would substantially increase the likelihood of recording and exploiting the Jovian DAM. The spatio-temporal analysis also revealed that emission frequencies are clustered in the CML vs Io plane and that emissions at low frequencies are more likely than in the high frequency range.

The statistical analysis on the Jovian DAM flux density distributions confirms the strong nature of the Jovian radiation with respect to the galactic background. The average flux densities are equal to $-159 \mathrm{~dB},-163 \mathrm{~dB}$ and $-167 \mathrm{~dB}$ for the Europa, Callisto and Ganymede case respectively. This implies that the mean relative power levels with respect to galactic noise range from 23 to $31 \mathrm{~dB}$ depending on the target moon. The peak values are substantially greater than the average levels. As an example, in the Europa case we could expect the strongest bursts to be $53 \mathrm{~dB}$ above galactic noise.

As a final remark, JUNO Waves data are provided in the form of power spectral densities and thus they are not suitable to address whether the Jovian DAM has stationarity properties. To address this issue, as future development we plan to analyze the properties of data acquired by terrestrial radio telescopes.

\section{ACKNOWLEDGMENT}

The authors would like to thank Dr. William S. Kurth and the JUNO Waves team at the University of Iowa for their invaluable support with the JUNO Waves data.

This research was in part supported by the Italian Space Agency through 'Attivita' scientifiche per JUICE fase C/D' under Contract ASI/INAF n. 2018-25-HH.0 and in part was carried out at the Jet Propulsion Laboratory, California Institute of Technology, under a contract with the National Aeronautics and Space Administration.

\section{REFERENCES}

[1] L. Bruzzone, J. J. Plaut, G. Alberti, D. D. Blankenship, F. Bovolo, B. A Campbell, D. Castelletti, Y. Gim, A. Ilisei, W. Kofman et al., "Jupiter icy moon explorer (juice): Advances in the design of the radar for icy moons (rime)," in Geoscience and Remote Sensing Symposium (IGARSS), 2015 IEEE International. IEEE, 2015, pp. 1257-1260.

[2] R. Croci, R. Seu, E. Flamini, and E. Russo, "The shallow radar (sharad) onboard the nasa mro mission," Proceedings of the IEEE, vol. 99, no. 5, pp. 794-807, 2011.

[3] L. J. Porcello, R. L. Jordan, J. S. Zelenka, G. Adams, R. Phillips, W. Brown, S. Ward, and P. Jackson, "The apollo lunar sounder radar system," Proceedings of the IEEE, vol. 62, no. 6, pp. 769-783, 1974.

[4] L. Carrer, C. Gerekos, and L. Bruzzone, "A multi-frequency radar sounder for lava tubes detection on the moon: Design, performance assessment and simulations," Planetary and Space Science, vol. 152, pp. 1-17, 2018.

[5] L. M. Prockter, "Ice in the solar system," Johns Hopkins APL technical digest, vol. 26, no. 2, pp. 175-188, 2005.

[6] L. Bruzzone, G. Alberti, C. Catallo, A. Ferro, W. Kofman, and R. Orosei, "Subsurface radar sounding of the jovian moon ganymede," Proceedings of the IEEE, vol. 99, no. 5, pp. 837-857, 2011.

[7] D. D. Blankenship, D. A. Young, W. B. Moore, and J. C. Moore, "Radar sounding of europa's subsurface properties and processes: The view from earth," Europa. University of Arizona Press, Tucson, AZ, 2009.

[8] B. Cecconi, S. Hess, A. Hérique, M. Santovito, D. Santos-Costa, P. Zarka, G. Alberti, D. Blankenship, J.-L. Bougeret, L. Bruzzone et al., "Natural radio emission of jupiter as interferences for radar investigations of the icy satellites of jupiter," Planetary and Space Science, vol. 61, no. 1, pp. 32-45, 2012.

[9] T. Carr, M. Desch, and J. Alexander, "Phenomenology of magnetospheric radio emissions," Physics of the jovian magnetosphere, pp. 226284, 1983

[10] A. Romero-Wolf, S. Vance, F. Maiwald, E. Heggy, P. Ries, and K. Liewer, "A passive probe for subsurface oceans and liquid water in jupiter's icy moons," Icarus, vol. 248, pp. 463-477, 2015.

[11] A. Romero-Wolf, D. M. Schroeder, P. Ries, B. G. Bills, C. Naudet, B. R. Scott, R. Treuhaft, and S. Vance, "Prospects of passive radio detection of a subsurface ocean on europa with a lander," Planetary and Space Science, vol. 129, pp. 118-121, 2016.

[12] C. Grima, D. D. Blankenship, and D. M. Schroeder, "Radar signal propagation through the ionosphere of europa," Planetary and Space Science, vol. 117, pp. 421-428, 2015.

[13] K. M. Scanlan, C. Grima, G. Steinbrügge, S. D. Kempf, D. A. Young, and D. D. Blankenship, "Geometric determination of ionospheric total electron content from dual frequency radar sounding measurements," Planetary and Space Science, vol. 178, p. 104696, 2019. 
[14] H. Cane, "Spectra of the non-thermal radio radiation from the galactic polar regions," Monthly Notices of the Royal Astronomical Society, vol. 189, no. 3, pp. 465-478, 1979.

[15] D. M. Schroeder, A. Romero-Wolf, L. Carrer, C. Grima, B. A. Campbell, W. Kofman, L. Bruzzone, and D. D. Blankenship, "Assessing the potential for passive radio sounding of europa and ganymede with rime and reason," Planetary and Space Science, vol. 134, pp. 52-60, 2016.

[16] Y. Aglyamov, D. M. Schroeder, and S. D. Vance, "Bright prospects for radar detection of europa's ocean," Icarus, vol. 281, pp. 334-337, 2017.

[17] P. Hartogh and Y. A. Ilyushin, "A passive low frequency instrument for radio wave sounding the subsurface oceans of the jovian icy moons: An instrument concept," Planetary and Space Science, vol. 130, pp. 30-39, 2016.

[18] C. Gerekos, L. Bruzzone, and M. Imai, "A coherent method for simulating active and passive radar sounding of the jovian icy moons," IEEE Transactions on Geoscience and Remote Sensing, 2019.

[19] E. Bigg, "Influence of the satellite io on jupiter's decametric emission," Nature, vol. 203, no. 4949, p. 1008, 1964.

[20] B. Burke and K. Franklin, "Observations of a variable radio source associated with the planet jupiter," Journal of Geophysical Research, vol. 60, no. 2, pp. 213-217, 1955.

[21] J.-L. Bougeret, "Very low frequency radio astronomy," Advances in Space Research, vol. 18, no. 11, pp. 35-41, 1996.

[22] W. Kurth, G. Hospodarsky, D. Kirchner, B. Mokrzycki, T. Averkamp, W. Robison, C. Piker, M. Sampl, and P. Zarka, "The juno waves investigation," Space Science Reviews, pp. 1-46, 2017.

[23] K. Kulpa, Signal processing in noise waveform radar. Artech House, 2013.

[24] S. R. Axelsson, "Noise radar for range/doppler processing and digital beamforming using low-bit adc," IEEE Transactions on Geoscience and Remote Sensing, vol. 41, no. 12, pp. 2703-2720, 2003.

[25] K. Kalousová, D. M. Schroeder, and K. M. Soderlund, "Radar attenuation in europa's ice shell: Obstacles and opportunities for constraining the shell thickness and its thermal structure," Journal of Geophysical Research: Planets, vol. 122, no. 3, pp. 524-545, 2017.

[26] C. Cook, Radar signals: An introduction to theory and application. Elsevier, 2012.

[27] N. J. Kasdin, "Discrete simulation of colored noise and stochastic processes and 1/f alpha; power law noise generation," Proceedings of the IEEE, vol. 83, no. 5, pp. 802-827, May 1995.

[28] W. Kurth and C. Piker, "Juno e/j/s/ss waves calibrated survey full resolution v1.0, jno-e/j/ss-wav-3-cdr-srvfull-v1.0, juno e/j/s/ss waves calibrated burst full resolution v1.0, jno-e/j/ss-wav-3-cdr-bstfull-v1.0," NASA Planetary Data System, 2012.

[29] M. Sampl, W. Macher, T. Oswald, D. Plettemeier, H. O. Rucker, and W. S. Kurth, "Juno model rheometry and simulation," Radio Science, vol. 51, no. 10, pp. 1627-1635, 2016.

[30] W. Kurth, B. Mauk, S. Elliott, D. Gurnett, G. Hospodarsky, O. Santolik, J. Connerney, P. Valek, F. Allegrini, G. Gladstone et al., "Whistler mode waves associated with broadband auroral electron precipitation at jupiter,' Geophysical Research Letters, vol. 45, no. 18, pp. 9372-9379, 2018.

[31] P. Zarka, "Auroral radio emissions at the outer planets: Observations and theories," Journal of Geophysical Research: Planets, vol. 103, no. E9, pp. $20159-20194,1998$.

[32] P. Zarka, M. Marques, C. Louis, V. Ryabov, L. Lamy, E. Echer, and B. Cecconi, "Radio emission from satellite-jupiter interactions (especially ganymede)," arXiv preprint arXiv:1709.04386, 2017.

[33] M. Imai, "Jupiter's decametric and hectometric radio emissions observed by cassini RPWS and voyager PRA," in PLANETARY RADIO EMISSIONS VII. Austrian Academy of Sciences Press, 2011. [Online]. Available: https://doi.org/10.1553/pre7s 167

[34] M. Imai, K. Imai, C. A. Higgins, and J. R. Thieman, "Comparison between cassini and voyager observations of jupiter's decametric and hectometric radio emissions," Journal of Geophysical Research: Space Physics, vol. 116, no. A12, 2011.

[35] H. Akaike, "Fitting autoregressive models for prediction," Annals of the institute of Statistical Mathematics, vol. 21, no. 1, pp. 243-247, 1969.

[36] C. H. Acton Jr, "Ancillary data services of nasa's navigation and ancillary information facility," Planetary and Space Science, vol. 44, no. 1, pp. 65-70, 1996.

[37] S. T. Peters, D. M. Schroeder, D. Castelletti, M. Haynes, and A. RomeroWolf, "In situ demonstration of a passive radio sounding approach using the sun for echo detection," IEEE Transactions on Geoscience and Remote Sensing, no. 99, pp. 1-12, 2018.
[38] M. Imai, W. S. Kurth, G. B. Hospodarsky, S. J. Bolton, J. E. Connerney, S. M. Levin, A. Lecacheux, L. Lamy, and P. Zarka, "Latitudinal beaming of jovian decametric radio emissions as viewed from juno and the nançay decameter array," Geophysical Research Letters, vol. 44, no. 10, pp. 4455-4462, 2017. 